\title{
PRIMES IN SHORT INTERVALS
}

\author{
Hugh L. MONTGOMERY ${ }^{1}$ \\ K. Soundararajan ${ }^{2}$ \\ Dedicated to Freeman Dyson, with best wishes on the occasion of his eightieth birthday.
}

\begin{abstract}
Contrary to what would be predicted on the basis of Cramér's model concerning the distribution of prime numbers, we develop evidence that the distribution of $\psi(x+H)-$ $\psi(x)$, for $0 \leq x \leq N$, is approximately normal with mean $\sim H$ and variance $\sim H \log N / H$, when $N^{\delta} \leq H \leq N^{1-\delta}$.
\end{abstract}

\section{INTRODUCTION}

Cramér [4] modeled the distribution of prime numbers by independent random variables $X_{n}$ (for $n \geq 3$ ) that take the value 1 ( $n$ is "prime") with probability $1 / \log n$ and take the value 0 ( $n$ is "composite") with probability $1-1 / \log n$. If $p_{n}$ denotes the $n^{\text {th }}$ prime number this model predicts that

$$
\lim _{N \rightarrow \infty} \frac{1}{N} \operatorname{card}\left\{n: 1 \leq n \leq N, p_{n+1}-p_{n}>c \log p_{n}\right\}=e^{-c}
$$

for all fixed positive real numbers $c$. Gallagher [6] showed that the above follows from Hardy \& Littlewood's [10, p. 61] quantitative version of the prime $k$-tuple conjecture: If $\mathcal{D}=\left\{d_{1}, d_{2}, \ldots, d_{k}\right\}$ is a set of $k$ distinct integers, then

$$
\sum_{n \leq x} \prod_{i=1}^{k} \Lambda\left(n+d_{i}\right)=(\mathfrak{S}(\mathcal{D})+o(1)) x
$$

as $x \rightarrow \infty$ where $\mathfrak{S}(\mathcal{D})$ is the singular series

$$
\mathfrak{S}(\mathcal{D})=\sum_{\substack{q_{1}, \ldots, q_{k} \\ 1 \leq q_{i}<\infty}}\left(\prod_{i=1}^{k} \frac{\mu\left(q_{i}\right)}{\phi\left(q_{i}\right)}\right) \sum_{\substack{a_{1}, \ldots, a_{k} \\ 1 \leq a_{i} \leq q_{i} \\\left(a_{i}, q_{i}\right)=1 \\ \sum a_{i} / q_{i} \in \mathbb{Z}}} e\left(\sum_{i=1}^{k} \frac{a_{i} d_{i}}{q_{i}}\right)
$$

1991 Mathematics Subject Classification. 11N05, 11M26, 11P45, 11N69.

Key words and phrases. primes, zeros of the Riemann zeta function, pair correlation, Cramér's model, random matrix theory.

${ }^{1}$ Research supported by NSF grants DMS-0070720 and DMS-0244660.

${ }^{2}$ Research supported by the American Institute of Mathematics and NSF grants. 
where $e(\theta)=e^{2 \pi i \theta}$. Hardy \& Littlewood showed that the right hand side above may be written more transparently as

$$
=\prod_{p}\left(1-\frac{1}{p}\right)^{-k}\left(1-\frac{\nu_{p}(\mathcal{D})}{p}\right)
$$

where $\nu_{p}(\mathcal{D})$ denotes the number of distinct residue classes modulo $p$ found among the members of $\mathcal{D}$. (See the remarks following the proof of Lemma 3 in $\S 2$.) Since $\nu_{p}(\mathcal{D})=k$ for all sufficiently large $p$, the product (3) is absolutely convergent. Hence its value is 0 if and only if there is a prime $p$ for which $\nu_{p}(\mathcal{D})=p$. Gallagher [6] showed that from (1) it follows that

$$
\int_{2}^{X}(\psi(x+\lambda \log x)-\psi(x))^{k} d x \sim m_{k}(\lambda) X(\log X)^{k}
$$

when $\lambda \asymp 1$. Here $m_{k}(\lambda)=\mathbb{E}\left(Y^{k}\right)$ is the $k^{\text {th }}$ moment of a Poisson random variable $Y$ with parameter $\lambda$, and $a \asymp b$ means that $a / b$ lies between two positive absolute constants. Thus the distribution of $\pi(x+h)-\pi(h)$ is approximately Poisson when $h \asymp \log X$, as predicted by the Cramér model.

In this paper we investigate the distribution of primes in longer intervals. Let $H=H(N)$ be a function of $N$ such that $H=o(N)$ and $H / \log N \rightarrow \infty$ as $N \rightarrow \infty$. The Cramér model predicts that the distribution of $\psi(n+H)-\psi(n)$ (for $n \leq N$ ) is approximately normal with mean $\sim H$ and variance $\sim H \log N$. Assuming a strong form of the Hardy-Littlewood conjecture (1) we will show that this prediction holds in the range where $H / \log N \rightarrow \infty$ and $\log H / \log N \rightarrow 0$ as $N \rightarrow \infty$. In the range $N^{\delta} \leq H \leq N^{1-\delta}$ we provide evidence showing that the Cramér model is incorrect, and conjecture instead that the distribution of $\psi(n+H)-\psi(n)$ is approximately normal with mean $\sim H$ and variance $\sim H \log (N / H)$.

When $h \asymp \log x$, the moments of $\psi(x+h)-\psi(x)$ and of $\psi(x+h)-\psi(x)-h$ are of the same order of magnitude. However, for larger $h$ one would expect that the moments of $\psi(x+h)-\psi(x)$ to be far larger than those of $\psi(x+h)-\psi(x)-h$. We obtain our conclusions on the distribution of $\psi(x+h)-\psi(x)$ by analyzing these latter, more delicate moments. To facilitate this study, we set $\Lambda_{0}(n)=\Lambda(n)-1$, with the result that

$$
\psi(x+h)-\psi(x)-h=\sum_{x<n \leq x+h} \Lambda_{0}(n) .
$$

Thus the main term is eliminated at the outset, which simplifies our calculations considerably. We recast (1) in an equivalent form that pertains to $\Lambda_{0}(n):$ If $d_{1}, \ldots, d_{k}$ are distinct integers, then

$$
\sum_{n \leq x} \prod_{i=1}^{k} \Lambda_{0}\left(n+d_{i}\right)=\left(\mathfrak{S}_{0}(\mathcal{D})+o(1)\right) x
$$

as $x \rightarrow \infty$ where $\mathfrak{S}_{0}(\mathcal{D})$ is related to $\mathfrak{S}(\mathcal{D})$ by the identities

$$
\begin{aligned}
\mathfrak{S}_{0}(\mathcal{D}) & =\sum_{\mathcal{J} \subseteq \mathcal{D}}(-1)^{\operatorname{card} \mathcal{J}} \mathfrak{S}(\mathcal{J}) \\
\mathfrak{S}(\mathcal{D}) & =\sum_{\mathcal{J} \subseteq \mathcal{D}} \mathfrak{S}_{0}(\mathcal{J})
\end{aligned}
$$


Here it is to be understood that $\mathfrak{S}_{0}(\emptyset)=\mathfrak{S}(\emptyset)=1$. One of the main steps in Gallagher's argument is to show that

$$
\sum_{\substack{d_{1}, \ldots, d_{k} \\ 1 \leq d_{i} \leq h \\ d_{i} \text { distinct }}} \mathfrak{S}(\mathcal{D}) \sim h^{k}
$$

as $h \rightarrow \infty$. There are $\left(\begin{array}{l}h \\ k\end{array}\right)$ subsets $\mathcal{D}$ under consideration, but each one occurs $k$ ! times in the above sum. Thus the above asserts that the mean value of $\mathfrak{S}(\mathcal{D})$ tends to 1 as $h \rightarrow \infty$. Correspondingly, we need to estimate the quantities

$$
R_{k}(h)=\sum_{\substack{d_{1}, \ldots, d_{k} \\ 1 \leq d_{i} \leq h \\ d_{i} \text { distinct }}} \mathfrak{S}_{0}(\mathcal{D})
$$

From (2) and (5) we see that

$$
\mathfrak{S}_{0}(\mathcal{D})=\sum_{\substack{q_{1}, \ldots, q_{k} \\ 1<q_{i}<\infty}}\left(\prod_{i=1}^{k} \frac{\mu\left(q_{i}\right)}{\phi\left(q_{i}\right)}\right) \sum_{\substack{a_{1}, \ldots, a_{k} \\ 1 \leq a_{i} \leq q_{i} \\\left(a_{i}, q_{i}\right)=1 \\ \sum a_{i} / q_{i} \in \mathbb{Z}}} e\left(\sum_{i=1}^{k} \frac{a_{i} d_{i}}{q_{i}}\right) .
$$

The task of estimating averages of this expression is quite challenging, but our burden is substantially lightened by work of Montgomery \& Vaughan [16] concerning a strikingly similar quantity. Let

$$
m_{k}(q ; h)=\sum_{n=1}^{q}\left(\sum_{\substack{m=1 \\(m+n, q)=1}}^{h} 1-h \phi(q) / q\right)^{k}
$$

be the $k^{\text {th }}$ centered moment of the number of reduced residues $(\bmod q)$ in an interval. Lemma 2 of Montgomery \& Vaughan asserts that

$$
m_{k}(q ; h)=q\left(\frac{\phi(q)}{q}\right)^{k} V_{k}(q ; h)
$$

where

$$
V_{k}(q ; h)=\sum_{\substack{d_{1}, \ldots, d_{k} \\ 1 \leq d_{i} \leq h}} \sum_{\substack{q_{1}, \ldots, q_{k} \\ 1<q_{i} \mid q}}\left(\prod_{i=1}^{k} \frac{\mu\left(q_{i}\right)}{\phi\left(q_{i}\right)}\right) \sum_{\substack{a_{1}, \ldots, a_{k} \\ 1 \leq a_{i} \leq q_{i} \\\left(a_{i}, q_{i}\right)=1 \\ \sum a_{i} / q_{i} \in \mathbb{Z}}} e\left(\sum_{i=1}^{k} \frac{a_{i} d_{i}}{q_{i}}\right) .
$$

When $k=1$, the conditions in the innermost sum cannot be fulfilled, and thus $V_{1}(q ; h)=0$. When $k=2$, the conditions in the innermost sum require that $q_{1}=q_{2}=a_{1}+a_{2}$. Thus

$$
V_{2}(q ; h)=\sum_{\substack{d \mid q \\ d>1}} \frac{\mu(d)^{2}}{\phi(d)^{2}} \sum_{\substack{a=1 \\(a, d)=1}}^{d}|E(a / d)|^{2}
$$


where

$$
E(\alpha)=\sum_{m=1}^{h} e(m \alpha)
$$

Montgomery \& Vaughan showed that

$$
V_{k}(q ; h) \ll_{k}(h q / \phi(q))^{k / 2}\left(1+O\left(h^{-1 /(7 k)}(q / \phi(q))^{2^{k}+k / 2}\right)\right)
$$

for each positive integer $k$. Unfortunately, this is not quite sharp enough for our present purposes, so our first job is to refine the above.

Theorem 1. In the above notation,

$$
V_{k}(q ; h)=\mu_{k} V_{2}(q ; h)^{k / 2}+O_{k}\left(h^{k / 2-1 /(7 k)}\left(\frac{q}{\phi(q)}\right)^{2^{k}+k / 2}\right)
$$

for every positive integer $k$, where $\mu_{k}=1 \cdot 3 \cdots(k-1)$ if $k$ is even, and $\mu_{k}=0$ if $k$ is odd.

Here the main term is the $k^{\text {th }}$ moment of a normal random variable with expectation 0 and variance $V_{2}(q ; h)$. We remark that the work of Granville \& Soundararajan [7] (see $\S 6 \mathrm{a})$ places restrictions on the uniformity (in $k$ ) with which (15) can possibly hold. With Theorem 1 in hand, we are able to estimate the $R_{k}(h)$.

Theorem 2. Let $h$ be an integer, $h>1$, and suppose that $R_{k}(h)$ is defined as in (8). Then

$$
R_{k}(h)=\mu_{k}(-h \log h+A h)^{k / 2}+O_{k}\left(h^{k / 2-1 /(7 k)+\varepsilon}\right)
$$

for any nonnegative integer $k$, where $A=2-C_{0}-\log 2 \pi$ and $C_{0}$ denotes Euler's constant.

For the smallest values of $k$, one can be more precise, since it is clear that $R_{0}(h)=1$, and that $R_{1}(h)=0$. Also, from (5) and (48) we know that

$$
R_{2}(h)=-h \log h+A h+O\left(h^{1 / 2+\varepsilon}\right) .
$$

From (6) it follows that the left hand side of (7) is

$$
\sum_{r=0}^{k}\left(\begin{array}{l}
k \\
r
\end{array}\right) R_{r}(h)(h-r)(h-r-1) \cdots(h-k+1) .
$$

Hence we obtain Gallagher's estimate (7) in the more precise form

$$
\sum_{\substack{d_{1}, \ldots, d_{k} \\
1 \leq d_{i} \leq h \\
d_{i} \text { distinct }}} \mathfrak{S}(\mathcal{D})=h^{k}-\left(\begin{array}{l}
k \\
2
\end{array}\right) h^{k-1} \log h+\left(\begin{array}{l}
k \\
2
\end{array}\right)\left(1-C_{0}-\log 2 \pi\right) h^{k-1}+O\left(h^{k-3 / 2+\varepsilon}\right) .
$$


We put

$$
M_{K}(N ; H)=\sum_{n=1}^{N}(\psi(n+H)-\psi(n)-H)^{K},
$$

and note that this is

$$
=\sum_{n=1}^{N}\left(\sum_{h=1}^{H} \Lambda_{0}(n+h)\right)^{K}=\sum_{\substack{h_{1}, \ldots, h_{K} \\ 1 \leq h_{i} \leq H}} \sum_{n=1}^{N} \prod_{i=1}^{K} \Lambda_{0}\left(n+h_{i}\right) .
$$

Here the $h_{i}$ are not necessarily distinct, but once the distinct values have been identified, and their multiplicities accounted for, we can appeal to (4). The quantities $R_{k}(h)$ arise in the main term. Thus from Theorem 2 we can derive an asymptotic estimate for the above, provided that the error term in (4) is sufficiently small and $H$ is not too large.

Theorem 3. Let $E_{k}(x ; \mathcal{D})$ be defined by the relation

$$
\sum_{n \leq x} \prod_{i=1}^{k} \Lambda\left(n+d_{i}\right)=\mathfrak{S}(\mathcal{D}) x+E_{k}(x ; \mathcal{D}),
$$

and suppose that

$$
E_{k}(x ; \mathcal{D}) \ll N^{1 / 2+\varepsilon}
$$

uniformly for $1 \leq k \leq K, 0 \leq x \leq N$, and distinct $d_{i}$ satisfying $1 \leq d_{i} \leq H$. Then

$$
\begin{aligned}
M_{K}(N ; H) & =\mu_{K} H^{K / 2} \int_{1}^{N}(\log x / H+B)^{K / 2} d x \\
& +O\left(N(\log N)^{K / 2} H^{K / 2}\left(\frac{H}{\log N}\right)^{-1 /(8 K)}+H^{K} N^{1 / 2+\varepsilon}\right)
\end{aligned}
$$

uniformly for $\log N \leq H \leq N^{1 / K}$, where $B=1-C_{0}-\log 2 \pi$ and $C_{0}$ denotes Euler's constant.

In the case $k=1$, the set $\mathcal{D}$ is a singleton, $\mathfrak{S}(\mathcal{D})=1$, and the hypothesis that $E_{1}(N ;\{1\}) \ll N^{1 / 2+\varepsilon}$ is equivalent to the Riemann Hypothesis (RH).

In place of $(20)$ if we assume only that $E_{k}(x ; \mathcal{D}) \ll E$ for some $E \geq N^{1 / 2+\varepsilon}$ then $(21)$ holds with the modified error term

$$
O\left(N(\log N)^{K / 2} H^{K / 2}\left(\frac{H}{\log N}\right)^{-1 /(8 K)}+H^{K} E\right) .
$$

We note that in deriving Theorem 3 from Theorem 2, we start with $h_{i}$ that are not necessarily distinct, and must reduce to distinct $d_{i}$, which is the reverse of the problem encountered in deriving Theorem 2 from Theorem 1, where we start with distinct $d_{i}$, and want to appeal to an estimate involving not necessarily distinct $m_{i}$.

Since the $\mu_{K}$ are the moments of a normal random variable with mean 0 and variance 1 we deduce from Theorem 3 the following Corollary. 
Corollary 1. Let $H=H(N)$ be a function of $N$ such that

$$
\frac{H}{\log N} \rightarrow+\infty, \quad \frac{\log H}{\log N} \rightarrow 0
$$

as $N \rightarrow \infty$. Assume that the hypothesis (20) holds for arbitrarily large $K$. Then the distribution of $\psi(n+H)-\psi(n)-H$ for $n \leq N$ is approximately normal with mean 0 and variance $H \log N$, in the sense that the proportion of $n \leq N$ for which $\psi(n+$ $H)-\psi(n)-H \leq c H \log N$ tends to $\Phi(c)$ as $N \rightarrow \infty$, uniformly for $|c| \leq C$. Here $\Phi(u)=\frac{1}{\sqrt{2 \pi}} \int_{-\infty}^{u} e^{-v^{2} / 2} d v$ is the cumulative distribution function of a normal random variable with mean 0 and variance 1 .

For larger $H$, Theorem 3 furnishes only a limited number of moments and we cannot deduce a distribution result. However we expect that the contributions of the $E_{k}(x ; \mathcal{D})$ cancel sufficiently so as not to overwhelm the main term:

Conjecture 1. For each positive integer $K$,

$$
M_{K}(N ; H)=\left(\mu_{K}+o(1)\right) N\left(H \log \frac{N}{H}\right)^{K / 2}
$$

uniformly for $(\log N)^{1+\delta} \leq H \leq N^{1-\delta}$.

This implies the weaker

Conjecture 2. Suppose that $(\log N)^{1+\delta} \leq H \leq N^{1-\delta}$. The distribution of $\psi(x+H)-\psi(x)$ for $0 \leq x \leq N$ is approximately normal with mean $H$ and variance $H \log N / H$.

Certainly Conjecture 2 does not hold when $H \asymp N$, but perhaps it holds whenever $H=o(N)$. It would be interesting to investigate more thoroughly what happens in this range.

Hardy \& Littlewood [10] provided heuristics that point toward the quantitative prime $k$-tuple conjecture (1). In $\S 4$ we argue in the same spirit to obtain indications in favor of Conjecture 1.

To obtain further support for our conjectures, we interpret the situation in terms of the zeros of the Riemann zeta function. We recall that Goldston \& Montgomery [8] showed that if $\mathrm{RH}$ is true, then the stronger form $(F(\alpha) \sim 1)$ of the Pair Correlation Conjecture as formulated by Montgomery [13] is equivalent to the case $K=2$ of the Conjecture above. In the same spirit, Chan [3] has shown (assuming RH) that Conjecture 1 is equivalent to the assertion that

$$
\int_{1}^{X}\left(\sum_{0<\gamma \leq T} \cos (\gamma \log x)\right)^{k} d x=\left(\mu_{k}+o(1)\right) X\left(\frac{T}{4 \pi} \log T\right)^{k / 2} .
$$

Viewed in this way, we see that the Pair Correlation Conjecture asserts that the variance of the sum

$$
\sum_{0<\gamma \leq T} \cos (\gamma \log x)
$$


is the same as it would be if it were a sum of uncorrelated random variables, and Conjecture 1 asserts that this same sum has the same normal distribution that it would have if the terms were independent random variables. In somewhat the same vein, Bogomolny \& Keating [1] used Hardy-Littlewood conjectures concerning primes to arrive at the $n$ level correlation function of zeros of the zeta function.

Freeman Dyson observed that the Pair Correlation Conjecture is analogous to known properties of the spacings of the eigenvalues of certain families of random matrices. We note that (22) has a similar analogue in random matrix theory. Let $U(N)$ denote the classical compact group of unitary $N \times N$ matrices. For $A \in U(N)$, let $e\left(\theta_{1}\right), \ldots, e\left(\theta_{N}\right)$ denote the eigenvalues of $A$. Rains [19] has observed that if $M$ is an integer, $|M| \geq N$, then the point $\left(M \theta_{1}, \ldots, M \theta_{N}\right)$ is exactly uniformly distributed in $\mathbb{T}^{N}$ as $A$ varies with respect to the Haar measure $d A$ on $U(N)$. It follows in particular that the distribution of

$$
\operatorname{Re} \operatorname{Trace} A^{M}=\sum_{n=1}^{N} \cos \left(2 \pi M \theta_{n}\right)
$$

is exactly the same as the distribution of

$$
\sum_{n=1}^{N} \cos 2 \pi X_{n}
$$

where where the $X_{n}$ are independent random variables, each one uniformly distributed on $[0,1]$. It follows by the Central Limit Theorem that this distribution tends toward a normal distribution with mean 0 and variance $N / 2$. By an easy calculation it can also be shown that the $k^{\text {th }}$ moment of this distribution is 0 if $k$ is odd, and is $\sim \mu_{k}(N / 2)^{k / 2}$ if $k$ is even.

As for numerical studies, Brent [2] has compiled evidence not only for (1) but also for the stronger hypothesis (20). Odlyzko [18] and Forrester \& Odlyzko [5] have found that the local distribution of the zeros of the zeta function fits well with predictions based on random matrix theory. The authors [15] have reported on numerical evidence in support of the conjectures. Finally, Chan [3; pp. 36, 49, 63] has assembled evidence in favor of (22).

Cramér's model suggests that

$$
\pi\left(x+(\log x)^{a}\right)-\pi(x) \sim(\log x)^{a-1}
$$

as $x \rightarrow \infty$ with $a$ fixed, $a>2$. This, however, is known to be false, since Maier [11] showed that

$$
\varlimsup_{x \rightarrow \infty} \frac{\pi\left(x+(\log x)^{a}\right)-\pi(x)}{(\log x)^{a-1}} \gtrless 1
$$

for any fixed $a>0$ (for general results of this nature see Granville \& Soundararajan [7]). Presumably (23) is valid for most $x$, and the exceptions discovered by Maier are quite rare. Indeed Selberg [18] showed that on RH, (23) holds if $a>2$ for almost all $x$, and Corollary 1 shows on hypothesis (20) that (23) holds if $a>1$ for almost all $x$. As for longer intervals, suppose that $\alpha$ is fixed, $0<\alpha<1$. Cramér's model would predict that 
$\psi\left(x+x^{\alpha}\right)-\psi(x)-x^{\alpha}$ is approximately normally distributed with mean 0 and variance $X^{\alpha} \log X$ as $x$ runs over the range $X \leq x \leq 2 X$. Our Conjecture 1 predicts normal distribution, but with a variance that is smaller by a factor of $1-\alpha$. Thus it seems that in this range, Cramér's model is not just occasionally inaccurate, but instead is actually inaccurate on average.

\section{Proof of Theorem 1}

Montgomery \& Vaughan [16] devised a useful basic inequality (their Lemma 1), which we now quote.

Lemma 1. Let $r_{1}, \ldots, r_{k}$ be squarefree integers, set $r=\left[r_{1}, \ldots, r_{k}\right]$, and suppose that any prime dividing $r$ divides at least two of the $r_{i}$. Then for any complex-valued functions $G_{1}, \ldots, G_{k}$ defined on $(0,1]$ we have

$$
\left|\sum_{\substack{b_{1}, \ldots, b_{k} \\ 1 \leq b_{i} \leq r_{i} \\ \sum b_{i} / r_{i} \in \mathbb{Z}}} \prod_{i=1}^{k} G_{i}\left(b_{i} / r_{i}\right)\right| \leq \frac{1}{r} \prod_{i=1}^{k}\left(r_{i} \sum_{b_{i}=1}^{r_{i}}\left|G_{i}\left(b_{i} / r_{i}\right)\right|^{2}\right)^{1 / 2} .
$$

Montgomery \& Vaughan [17] have derived several variants of the above; an exposition of such variants is found in Chapter 8 of Montgomery [14]. For our present purposes a different type of variant is useful.

Lemma 2. Let $q_{1}, \ldots, q_{k}$ be squarefree integers, each one strictly greater than 1 , and put $d=\left[q_{1}, \ldots, q_{k}\right]$. Let $G$ be a complex-valued function defined on $(0,1)$, and suppose that $G_{0}$ is a nondecreasing function on the positive integers such that

$$
\sum_{a=1}^{q-1}|G(a / q)|^{2} \leq q G_{0}(q)
$$

for all squarefree integers $q>1$. Then

$$
\left|\sum_{\substack{a_{1}, \ldots, a_{k} \\ 0<a_{i}<q_{i} \\ \sum a_{i} / q_{i} \in \mathbb{Z}}} \prod_{i=1}^{k} G\left(a_{i} / q_{i}\right)\right| \leq \frac{1}{d} \prod_{i=1}^{k} q_{i} G_{0}\left(q_{i}\right)^{1 / 2} .
$$

Proof. We write $q_{i}=r_{i} s_{i}$ where the $s_{i}$ are pairwise relatively prime and any prime dividing $\left[r_{1}, \ldots, r_{k}\right]$ divides at least two of the $r_{i}$. That is, $r_{i}=\left(q_{i}, \prod_{j \neq i} q_{j}\right)$. Clearly $d=r s_{1} \cdots s_{k}$ where $r=\left[r_{1}, \ldots, r_{k}\right]$. The condition $\sum a_{i} / q_{i} \in \mathbb{Z}$ forces $s_{i} \mid a_{i}$ for all $i$. Hence, on writing $a_{i}=s_{i} b_{i}$, we find that the left hand side above is

$$
=\left|\sum_{\substack{b_{1}, \ldots, b_{k} \\ 0<b_{i}<r_{i} \\ \sum b_{i} / r_{i} \in \mathbb{Z}}} \prod_{i=1}^{k} G\left(b_{i} / r_{i}\right)\right| .
$$


If there is an $i$ for which $r_{i}=1$, then the conditions in the above sum cannot be fulfilled, the sum is empty, and there is nothing to prove. Thus we may assume that $r_{i}>1$ for all $i$. In Lemma 1 we take $G_{i}(x)=G(x)$ for $0<x<1$, and $G(1)=0$. Thus by Lemma 1 and the hypothesis (24), the above is

$$
\leq \frac{1}{r} \prod_{i=1}^{k}\left(r_{i} \sum_{b_{i}=1}^{r_{i}-1} r_{i}\left|G\left(b_{i} / r_{i}\right)\right|^{2}\right)^{1 / 2} \leq \frac{1}{r} \prod_{i=1}^{k}\left(r_{i}^{2} G_{0}\left(r_{i}\right)\right)^{1 / 2}
$$

Since $G$ is nondecreasing, the above is

$$
\leq \frac{1}{r} \prod_{i=1}^{k}\left(r_{i}^{2} G_{0}\left(q_{i}\right)\right)^{1 / 2}=\frac{1}{d} \prod_{i=1}^{k}\left(q_{i}^{2} G_{0}\left(q_{i}\right)\right)^{1 / 2}
$$

as desired.

We now begin the main body of the proof of Theorem 1 . We take $k$ to be fixed, so that the dependence of implicit constants on $k$ is suppressed. If $k$ is odd, then the desired estimate is already found in (18) of Montgomery \& Vaughan [16]. Thus we may assume that $k$ is even. From (12) and (14) it is clear that

$$
V_{k}(q ; h)=\sum_{\substack{q_{1}, \ldots, q_{k} \\ 1<q_{i} \mid q}}\left(\prod_{i=1}^{k} \frac{\mu\left(q_{i}\right)}{\phi\left(q_{i}\right)}\right) \sum_{\substack{a_{1}, \ldots, a_{k} \\ 1 \leq a_{i} \leq q_{i} \\\left(a_{i}, q_{i}\right)=1 \\ \sum a_{i} / q_{i} \in \mathbb{Z}}} \prod_{i=1}^{k} E\left(a_{i} / q_{i}\right)
$$

In Lemmas 7 and 8 of Montgomery \& Vaughan [16], it is shown that all contributions to the above are

$$
\ll h^{k / 2-1 /(7 k)}\left(\frac{q}{\phi(q)}\right)^{2^{k}+k / 2},
$$

except for those terms for which the $q_{i}$ are equal in pairs, with no further equalities among the $q_{i}$. There are $(k-1)(k-3) \cdots 3 \cdot 1=\mu_{k}$ ways in which this pairing can occur. Take the pairing to be $q_{i}=q_{k / 2+i}$, and set $b_{i}=a_{i}+a_{k / 2+i}$. Thus the terms that remain to be estimated are precisely

$$
\mu_{k} \sum_{\substack{q_{1}, \ldots, q_{k / 2} \\ 1<q_{i} \mid q \\ q_{i} \text { distinct }}} \prod_{i=1}^{k / 2} \frac{\mu\left(q_{i}\right)^{2}}{\phi\left(q_{i}\right)^{2}} \sum_{\substack{b_{1}, \ldots, b_{k / 2} \\ 1 \leq b_{i} \leq q_{i} \\ \sum b_{i} / q_{i} \in \mathbb{Z}}} \prod_{i=1}^{k / 2} J\left(b_{i}, q_{i}\right)
$$

where

$$
J(b, r)=\sum_{\substack{a=1 \\(a, r)=1 \\(b-a, r)=1}}^{r} E\left(\frac{a}{r}\right) E\left(\frac{b-a}{r}\right)
$$


First we show that the condition that the $q_{i}$ should be distinct in (25) can be dropped. To see this, put $F(\alpha)=\min (h, 1 /\|\alpha\|)$ where $\|\theta\|=\min _{n \in \mathbb{Z}}|\theta-n|$ is the distance from $\theta$ to the nearest integer. Thus

$$
|E(\alpha)| \leq F(\alpha)
$$

for all $\alpha$. Let $\mathcal{Q}$ denote the set of those $k$-tuples $\left(q_{1}, \ldots, q_{k}\right)$ such that $1<q_{i} \mid q$ for all $i$, and with the property that among the $q_{i}$ there are three or more of them that are equal. In proving their Lemma 8 (see the treatment of $T_{3}$ ), Montgomery \& Vaughan [16] establish that

$$
\sum_{\boldsymbol{q} \in \mathcal{Q}}\left(\prod_{i=1}^{k} \frac{\left|\mu\left(q_{i}\right)\right|}{\phi\left(q_{i}\right)}\right) \sum_{\substack{a_{1}, \ldots, a_{k} \\ 1 \leq a_{i} \leq q_{i} \\\left(a_{i}, q_{i}\right)=1 \\ \sum a_{i} / q_{i} \in \mathbb{Z}}} \prod_{i=1}^{k} F\left(a_{i} / q_{i}\right) \ll h^{k / 2-1 /(7 k)}\left(\frac{q}{\phi(q)}\right)^{2^{k}+k / 2} .
$$

Since this majorizes the difference between (25) and

$$
\mu_{k} \sum_{\substack{q_{1}, \ldots, q_{k / 2} \\ 1<q_{i} \mid q}} \prod_{i=1}^{k / 2} \frac{\mu\left(q_{i}\right)^{2}}{\phi\left(q_{i}\right)^{2}} \sum_{\substack{b_{1}, \ldots, b_{k / 2} \\ 1 \leq b_{i} \leq q_{i} \\ \sum b_{i} / q_{i} \in \mathbb{Z}}} \prod_{i=1}^{k / 2} J\left(b_{i}, q_{i}\right)
$$

it follows that we can continue with the above expression. Suppose that $0<b_{i}<q_{i}$ for exactly $j$ values of $i$, and that $b_{i}=q_{i}$ for the remaining $k / 2-j$ values of $i$. Since there are $\left(\begin{array}{c}k / 2 \\ j\end{array}\right)$ ways of choosing the $j$ indicies, we see that the above is

$$
\mu_{k} \sum_{j=0}^{k / 2}\left(\begin{array}{c}
k / 2 \\
j
\end{array}\right) V_{2}(q ; h)^{k / 2-j} W_{j}(q ; h)
$$

where $W_{0}(q ; h)=1$ and

$$
W_{j}(q ; h)=\sum_{\substack{q_{1}, \ldots, q_{j} \\ 1<q_{i} \mid q}} \prod_{i=1}^{j} \frac{\mu\left(q_{i}\right)^{2}}{\phi\left(q_{i}\right)^{2}} \sum_{\substack{b_{1}, \ldots, b_{j} \\ 0<b_{i}<q_{i} \\ \sum b_{i} / q_{i} \in \mathbb{Z}}} \prod_{i=1}^{j} J\left(b_{i}, q_{i}\right)
$$

Here the term $j=0$ gives the desired main term. Thus it remains to show that the other terms are smaller.

To prepare for an application of Lemma 2, we estimate $J(b, r)$. By $(27)$ we see that if $0<b \leq r / 2$ and $r<h$, then

$$
\begin{aligned}
J(b, r) & \ll \sum_{b / 2<a<b} \frac{r^{2}}{b(b-a)}+\sum_{b<a \leq 3 b / 2} \frac{r^{2}}{b(a-b)}+\sum_{3 b / 2<a \leq 2 r / 3} \frac{r^{2}}{a^{2}} \\
& \ll \frac{r^{2}}{b} \log 2 b .
\end{aligned}
$$


Here half the ranges of $a$ have been omitted, since by symmetry they contribute the same amount as the listed sums. Similarly, if $0<b \leq r / h$ and $r \geq h$, then

$$
J(b, r) \ll \sum_{0<a \leq 2 r / h} h^{2}+\sum_{2 r / h<a \leq r / 2} \frac{r^{2}}{a^{2}} \ll r h .
$$

Finally, if $r / h<b \leq r / 2$ and $r \geq h$, then

$$
\begin{aligned}
J(b, r) & \ll \sum_{b / 2<a \leq b-r / h} \frac{r^{2}}{b(b-a)}+\sum_{b-r / h<a<b} \frac{r h}{a}+\sum_{b<a \leq b+r / h} \frac{r h}{a} \\
& +\sum_{b+r / h<a \leq 4 b / 3} \frac{r^{2}}{b(a-b)}+\sum_{4 b / 3<a \leq 2 r / 3} \frac{r^{2}}{a^{2}} \\
& \ll \frac{r^{2}}{b} \log (2 b h / r) .
\end{aligned}
$$

From (31) we see that if $r<h$, then

$$
\sum_{0<b<r} J(b, r)^{2} \ll r^{4}
$$

and from (32) and (33) we see that if $r \geq h$, then

$$
\sum_{0<b<r} J(b, r)^{2} \ll r^{3} h
$$

Altogether,

$$
\sum_{0<b<r} J(b, r)^{2} \ll r^{3} \min (r, h) .
$$

On taking $G_{0}(r)=C h r^{2}$ in Lemma 2, we find that

$$
\sum_{\substack{b_{1}, \ldots, b_{j} \\ 0<b_{i}<q_{i} \\ \sum b_{i} / q_{i} \in \mathbb{Z}}} \prod_{i=1}^{j} J\left(b_{i}, q_{i}\right) \ll \frac{1}{d} \prod_{i=1}^{j}\left(q_{i}^{2} h^{1 / 2}\right),
$$

and hence

$W_{j}(q ; h) \ll h^{j / 2} \sum_{d \mid q} \frac{1}{d}\left(\sum_{r \mid d} \frac{\mu(r)^{2} r^{2}}{\phi(r)^{2}}\right)^{j}=h^{j / 2} \prod_{p \mid q}\left(1+\frac{1}{p}\left(1+\frac{p^{2}}{(p-1)^{2}}\right)^{j}\right) \ll h^{j / 2}\left(\frac{q}{\phi(q)}\right)^{2^{j}}$.

To apply this in $(29)$, we need also a bound for $V_{2}(q ; h)$. To this end we note that

$$
\begin{aligned}
V_{2}(q ; h) & \leq \sum_{d \mid q} \frac{\mu(d)^{2}}{\phi(d)^{2}} \sum_{a=1}^{d-1} F(a / d)^{2} \ll h \sum_{d \mid q} \frac{\mu(d)^{2} d}{\phi(d)^{2}} \\
& =h \prod_{p \mid q}\left(1+\frac{p}{(p-1)^{2}}\right) \ll h \frac{q}{\phi(q)} .
\end{aligned}
$$


(By a different method it can be shown that $V_{2}(q ; h) \leq h q / \phi(q)$. See Hausman \& Shapiro [11] and (3) of Montgomery \& Vaughan [16].) From (30) we see that $W_{1}(q ; h)=0$, since the inner sum is empty. On applying the above estimates for $2 \leq j \leq k / 2$, we see that the expression (29) is

$$
\mu_{k} V_{2}(q ; h)^{k / 2}+O\left(h^{k / 2-1}(q / \phi(q))^{2^{k / 2}}\right) .
$$

Here the error term is majorized by that in (15), so the proof is complete.

\section{Proof of Theorem 2}

We begin with two lemmas.

Lemma 3. (Hardy-Littlewood) Let

$$
A\left(q_{1}, \ldots, q_{k}\right)=\sum_{\substack{a_{1}, \ldots, a_{k} \\ 1 \leq a_{i} \leq q_{i} \\\left(a_{i}, q_{i}\right)=1 \\ \sum a_{i} / q_{i} \in \mathbb{Z}}} e\left(\sum_{i=1}^{k} \frac{d_{i} a_{i}}{q_{i}}\right) .
$$

If $q_{i}=q_{i}^{\prime} q_{i}^{\prime \prime}$ with $\left(\prod q_{i}^{\prime}, \prod q_{i}^{\prime \prime}\right)=1$, then

$$
A\left(q_{1}, \ldots, q_{k}\right)=A\left(q_{1}^{\prime}, \ldots, q_{k}^{\prime}\right) A\left(q_{1}^{\prime \prime}, \ldots, q_{k}^{\prime \prime}\right) .
$$

For any prime number $p$,

$$
\sum_{\substack{q_{1}, \ldots, q_{k} \\ q_{i} \mid p}} \prod_{i=1}^{k} \frac{\mu\left(q_{i}\right)}{\phi\left(q_{i}\right)} A\left(q_{1}, \ldots, q_{k}\right)=\left(1-\frac{1}{p}\right)^{-k}\left(1-\frac{\nu_{p}(\mathcal{D})}{p}\right)
$$

where $\nu_{p}(\mathcal{D})$ is the number of distinct residue classes modulo $p$ found among the members of $\mathcal{D}=\left\{d_{1}, \ldots, d_{k}\right\}$. Finally,

$$
\sum_{\substack{q_{1}, \ldots, q_{k} \\ 1 \leq q_{i}<\infty}} \prod_{i=1}^{k} \frac{\mu\left(q_{i}\right)^{2}}{\phi\left(q_{i}\right)}\left|A\left(q_{1}, \ldots, q_{k}\right)\right|<\infty
$$

Proof. We follow the argument of Hardy \& Littlewood [10, pp. 56-61], but with some helpful amplifications. We write

$$
\frac{a_{i}}{q_{i}} \equiv \frac{a_{i}^{\prime}}{q_{i}^{\prime}}+\frac{a_{i}^{\prime \prime}}{q_{i}^{\prime \prime}} \quad(\bmod 1)
$$

By the Chinese Remainder Theorem, each reduced residue $a_{i}$ modulo $q_{i}$ corresponds to a pair $a_{i}^{\prime}, a_{i}^{\prime \prime}$ of reduced residues modulo $q_{i}^{\prime}$ and $q_{i}^{\prime \prime}$, respectively. Also, $\sum a_{i} / q_{i} \in \mathbb{Z}$ if and only if $\sum a_{i}^{\prime} / q_{i}^{\prime} \in \mathbb{Z}$ and $\sum a_{i}^{\prime \prime} / q_{i}^{\prime \prime} \in \mathbb{Z}$. This gives (35). 
If each $q_{i}$ is either 1 or $p$, then

$$
\sum_{r=1}^{p} e\left(\sum_{i=1}^{k} \frac{a_{i} r}{q_{i}}\right)= \begin{cases}p & \text { if } \sum_{i=1}^{k} a_{i} / q_{i} \in \mathbb{Z} \\ 0 & \text { otherwise }\end{cases}
$$

Thus the left hand side of (36) is

$$
\begin{aligned}
& =\frac{1}{p} \sum_{r=1}^{p} \sum_{\substack{q_{1}, \ldots, q_{k} \\
q_{i} \mid p}} \prod_{i=1}^{k} \frac{\mu\left(q_{i}\right)}{\phi\left(q_{i}\right)} \sum_{\substack{a_{1}, \ldots, a_{k} \\
1 \leq a_{i} \leq q_{i} \\
\left(a_{i}, q_{i}\right)=1}} e\left(\sum_{i=1}^{k} \frac{a_{i}\left(d_{i}-r\right)}{q_{i}}\right) \\
& =\frac{1}{p} \sum_{r=1}^{p} \prod_{i=1}^{k}\left(1-\frac{1}{p-1} \sum_{0<a<p} e\left(\frac{a\left(d_{i}-r\right)}{p}\right)\right) .
\end{aligned}
$$

Here the innermost sum is $p-1$ or -1 , according as $r \equiv d_{i}(\bmod p)$, or not. Thus if $r \equiv d_{i}$ $(\bmod p)$, then this factor of the product is 0 . There are $\nu_{p}(\mathcal{D})$ such values of $r$. For the remaining $p-\nu_{p}(\mathcal{D})$ values of $r$, each factor of the product is $p /(p-1)$. Hence the above is

$$
=\frac{p-\nu_{p}(\mathcal{D})}{p}\left(\frac{p}{p-1}\right)^{k}
$$

which gives (36).

From (35) we see that

$$
\sum_{\substack{q_{1}, \ldots, q_{k} \\ q_{i} \mid Q}} \prod_{i=1}^{k} \frac{\mu\left(q_{i}\right)^{2}}{\phi\left(q_{i}\right)}\left|A\left(q_{1}, \ldots, q_{k}\right)\right|=\prod_{p \mid Q}\left(\sum_{\substack{q_{1}, \ldots, q_{k} \\ q_{i} \mid p}} \prod_{i=1}^{k} \frac{1}{\phi\left(q_{i}\right)}\left|A\left(q_{1}, \ldots, q_{k}\right)\right|\right) .
$$

Put $D=\prod_{i<j}\left(d_{j}-d_{i}\right)$. For primes $p \mid D$ we make no attempt to simplify the above expression. However, there are only finitely many such primes, and for $p \nmid D$, the $d_{i}$ are distinct modulo $p$. For such primes we evaluate the factor more explicitly. Let $\mathcal{J} \subseteq$ $\{1, \ldots, k\}$ with $j=$ card $\mathcal{J}$, suppose that $q_{i}=p$ for $i \in \mathcal{J}, q_{i}=1$ for $i \notin \mathcal{J}$, and that the $d_{i}$ are distinct modulo $p$. Then

$$
A\left(q_{1}, \ldots, q_{k}\right)=\frac{1}{p} \sum_{r=1}^{p} \sum_{\substack{a_{1}, \ldots, a_{k} \\ 1 \leq a_{i} \leq q_{i} \\\left(a_{i}, q_{i}\right)=1}} e\left(\frac{a_{i}\left(d_{i}-r\right)}{q_{i}}\right)=\frac{1}{p} \sum_{r=1}^{p} \prod_{i=1}^{k}\left(\sum_{\substack{1 \leq a_{i} \leq q_{i} \\\left(a_{i}, q_{i}\right)=1}} e\left(\frac{a_{i}\left(d_{i}-r\right)}{q_{i}}\right)\right) .
$$

If $i \notin \mathcal{J}$, then the innermost sum is 1 for all $r$. If $i \in \mathcal{J}$, then the innermost sum is $p-1$ if $r \equiv d_{i}(\bmod p)$, and -1 otherwise. Thus there are $j$ values of $r$ for which one factor is $p-1, j-1$ factors are -1 , and all other factors are 1 . For the remaining $p-j$ values of $r$, there are $j$ factors that are -1 and the remaining factors are 1 . Thus the above is

$$
=\frac{1}{p}\left(j(p-1)(-1)^{j-1}+(p-j)(-1)^{j}\right)=(-1)^{j-1}(j-1) .
$$


Hence $\left|A\left(q_{1}, \ldots, q_{k}\right)\right|=|j-1|$, so it follows that the expression (38) is

$$
\prod_{\substack{p|Q \\
p| D}}\left(\sum_{\substack{q_{1}, \ldots, q_{i} \mid p}} \prod_{\substack{q_{k} \\
i=1}}^{k} \frac{1}{\phi\left(q_{i}\right)}\left|A\left(q_{1}, \ldots, q_{k}\right)\right|\right) \times \prod_{\substack{p \mid Q \\
p \nmid D}}\left(1+\sum_{j=2}^{k}\left(\begin{array}{c}
k \\
j
\end{array}\right) \frac{j-1}{(p-1)^{j}}\right) .
$$

Since this last product converges when extended over all primes, we have (37), and the proof is complete.

From (35) and (36) we see that

$$
\sum_{\substack{q_{1}, \ldots, q_{k} \\ q_{i} \mid Q}} \prod_{i=1}^{k} \frac{\mu\left(q_{i}\right)}{\phi\left(q_{i}\right)} A\left(q_{1}, \ldots, q_{k}\right)=\prod_{p \mid Q}\left(1-\frac{1}{p}\right)^{-k}\left(1-\frac{\nu_{p}(\mathcal{D})}{p}\right)
$$

for any positive integer $Q$. By (37) it follows that

$$
\mathfrak{S}(\mathcal{D})=\lim _{y \rightarrow \infty} \sum_{\substack{q_{1}, \ldots, q_{k} \\ p \mid q_{i} \Rightarrow p \leq y}} \prod_{i=1}^{k} \frac{\mu\left(q_{i}\right)}{\phi\left(q_{i}\right)} A\left(q_{1}, \ldots, q_{k}\right)
$$

which by (39) is

$$
=\lim _{y \rightarrow \infty} \prod_{p \leq y}\left(1-\frac{1}{p}\right)^{-k}\left(1-\frac{\nu_{p}(\mathcal{D})}{p}\right) .
$$

Thus the expressions (2) and (3) are equal.

Suppose that $1 \leq d_{i} \leq h$ for all $i$. Then $\nu_{p}(\mathcal{D})=k$ for all primes $p>h$, and thus if $y \geq h$, then

$$
\prod_{p>y}\left(1-\frac{1}{p}\right)^{-k}\left(1-\frac{\nu_{p}(\mathcal{D})}{p}\right)=\prod_{p>y}\left(1+O_{k}\left(\frac{1}{p^{2}}\right)\right)=1+O_{k}\left(\frac{1}{y \log y}\right) .
$$

Since $\nu_{p}(\mathcal{D}) \geq 1$ for all $p$, it is evident that

$$
\prod_{p \leq h}\left(1-\frac{1}{p}\right)^{-k}\left(1-\frac{\nu_{p}(\mathcal{D})}{p}\right) \ll_{k}(\log h)^{k-1} .
$$

On combining this with (40), we see that

$$
\mathfrak{S}(\mathcal{D}) \ll(\log h)^{k-1} .
$$

From (5) it follows additionally that

$$
\mathfrak{S}_{0}(\mathcal{D}) \ll(\log h)^{k-1} .
$$


From (39)-(41) we see that if $1 \leq d_{i} \leq h$ for all $i$ and $y \geq h$, then

$$
\mathfrak{S}(\mathcal{D})=\sum_{\substack{q_{1}, \ldots, q_{k} \\ p \mid q_{i} \Rightarrow p \leq y}} \prod_{i=1}^{k} \frac{\mu\left(q_{i}\right)}{\phi\left(q_{i}\right)} A\left(q_{1}, \ldots, q_{k}\right)+O_{k}\left(\frac{(\log y)^{k-2}}{y}\right) .
$$

By combining this with (5), we see also that

$$
\mathfrak{S}_{0}(\mathcal{D})=\sum_{\substack{q_{1}, \ldots, q_{k} \\ q_{i}>1 \\ p \mid q_{i} \Rightarrow p \leq y}} \prod_{i=1}^{k} \frac{\mu\left(q_{i}\right)}{\phi\left(q_{i}\right)} A\left(q_{1}, \ldots, q_{k}\right)+O_{k}\left(\frac{(\log y)^{k-2}}{y}\right) .
$$

Lemma 4. Let $E(\alpha)$ be defined as in (14). If $q$ is divisible by every prime number $p \leq h^{2}$, then

$$
\sum_{d \mid q} \frac{\mu(d)^{2}}{\phi(d)^{2}}\left(\sum_{\substack{a=1 \\(a, d)=1}}^{d}|E(a / d)|^{2}-\phi(d) h\right)=h^{2}-h \log h+B h+O\left(h^{1 / 2+\varepsilon}\right)
$$

where $B=1-C_{0}-\log 2 \pi$ and $C_{0}$ denotes Euler's constant.

Proof. Since $|E(\alpha)|^{2}=\sum_{|m| \leq h}(h-|m|) e(m \alpha)$, it follows that

$$
\sum_{\substack{a=1 \\(a, d)=1}}^{d}|E(a / d)|^{2}=\sum_{|m| \leq h}(h-|m|) c_{d}(m)
$$

where $c_{d}(m)$ is Ramanujan's sum. Now $c_{d}(0)=\phi(d)$, and $c_{d}(-m)=c_{d}(m)$, so the above is

$$
\phi(d) h+2 \sum_{m=1}^{h}(h-m) c_{d}(m) .
$$

Hence the left hand side of (46) is

$$
=2 \sum_{m=1}^{h}(h-m) \sum_{d \mid q} \frac{\mu(d)^{2}}{\phi(d)^{2}} c_{d}(m)
$$

Here the sum over $d$ is

$$
\prod_{p \mid q}\left(1+\frac{c_{p}(m)}{(p-1)^{2}}\right)=\prod_{\substack{p|q \\ p| m}}\left(1+\frac{1}{p-1}\right) \prod_{\substack{p \mid q \\ p \nmid m}}\left(1-\frac{1}{(p-1)^{2}}\right) .
$$

Since $q$ is divisible by every prime $p \leq h^{2}$, the above is

$$
=\prod_{p \mid m}\left(1+\frac{1}{p-1}\right) \prod_{p \nmid m}\left(1-\frac{1}{(p-1)^{2}}\right)+O\left(1 / h^{2}\right) .
$$


Here the main term is

$$
\prod_{p \mid m}\left(1-\frac{1}{p}\right)^{-2}\left(1-\frac{1}{p}\right) \times \prod_{p \nmid m}\left(1-\frac{1}{p}\right)^{-2}\left(1-\frac{2}{p}\right)=\mathfrak{S}(\{0, m\}) .
$$

Goldston [7] has shown that

$$
2 \sum_{m=1}^{h}(h-m) \mathfrak{S}(\{0, m\})=h^{2}-h \log h+B h+O\left(h^{1 / 2+\varepsilon}\right),
$$

so we have the stated estimate.

It is worth noting that (47) can also be written in the form

$$
\sum_{\substack{d_{1}, d_{2} \\ 1 \leq d_{i} \leq h \\ d_{1} \neq d_{2}}} \mathfrak{S}\left(\left\{d_{1}, d_{2}\right\}\right)=h^{2}-h \log h+B h+O\left(h^{1 / 2+\varepsilon}\right) .
$$

The term $d=1$ contributes $h^{2}-h$ to the left hand side of (46). Thus if $q$ is divisible by every prime not exceeding $h^{2}$, then

$$
\sum_{\substack{d \mid q \\ d>1}} \frac{\mu(d)^{2}}{\phi(d)^{2}}\left(\sum_{\substack{a=1 \\(a, d)=1}}^{d}|E(a / d)|^{2}-\phi(d) h\right)=-h \log h+A h+O\left(h^{1 / 2+\varepsilon}\right)
$$

where $A=2-C_{0}-\log 2 \pi$.

We now begin the main body of the proof of Theorem 2. We apply (45) with $y=h^{k+1}$, and set $Q=\prod_{p \leq y} p$. Thus

$$
R_{k}(h)=\sum_{\substack{q_{1}, \ldots, q_{k} \\ 1<q_{i} \\ q_{i} \mid Q}} \prod_{i=1}^{k} \frac{\mu\left(q_{i}\right)}{\phi\left(q_{i}\right)} S\left(q_{1}, \ldots, q_{k} ; h\right)+O(1)
$$

where

$$
S\left(q_{1}, \ldots, q_{k} ; h\right)=\sum_{\substack{d_{1}, \ldots, d_{k} \\ 1 \leq d_{i} \leq h \\ d_{i} \text { distinct }}} \sum_{\substack{a_{1}, \ldots, a_{k} \\ 1 \leq a_{i} \leq q_{i} \\\left(a_{i}, q_{i}\right)=1 \\ \sum a_{i} / q_{i} \in \mathbb{Z}}} e\left(\sum_{i=1}^{k} \frac{a_{i} d_{i}}{q_{i}}\right)
$$

By comparing (50) with (12) we find that if the condition that the $d_{i}$ should be distinct were omitted, then the main term in (50) would be exactly $V_{k}(Q ; h)$. The bulk of our 
argument is devoted to an effort to remove this condition. Put $\delta_{i, j}=1$ if $d_{i}=d_{j}, \delta_{i, j}=0$ otherwise. Thus

$$
\prod_{1 \leq i<j \leq k}\left(1-\delta_{i, j}\right)= \begin{cases}1 & \text { if the } d_{i} \text { are distinct; } \\ 0 & \text { otherwise }\end{cases}
$$

When the left hand side above is expanded, we obtain a linear combination of products of the $\delta$ symbols. Let $\Delta$ denote such a product, and $|\Delta|$ the number of factors in the product. We define an equivalence relation on these $\delta$-products by setting $\Delta_{1} \sim \Delta_{2}$ if $\Delta_{1}$ and $\Delta_{2}$ have the same value for all choices of $d_{1}, \ldots, d_{k}$. For example, $\delta_{1,2} \delta_{1,3} \sim \delta_{1,2} \delta_{2,3} \sim$ $\delta_{1,2} \delta_{1,3} \delta_{2,3}$. Given a partition $\mathcal{P}=\left\{\mathcal{S}_{1}, \ldots, \mathcal{S}_{M}\right\}$ of the set $\{1, \ldots, k\}$, let

$$
\Delta_{\mathcal{P}}=\prod_{m=1}^{M} \prod_{\substack{i<j \\ i \in \mathcal{S}_{m} \\ j \in \mathcal{S}_{m}}} \delta_{i, j}
$$

We see easily that every equivalence class of $\delta$-products contains a unique $\Delta_{\mathcal{P}}$. Thus we have a bijective correspondence between equivalence classes of $\delta$-products and partitions of $\{1, \ldots, k\}$. For a partition $\mathcal{P}$, put

$$
w(\mathcal{P})=\sum_{\Delta \sim \Delta_{\mathcal{P}}}(-1)^{|\Delta|} .
$$

Thus

$$
\prod_{1 \leq i<j \leq k}\left(1-\delta_{i, j}\right)=\sum_{\mathcal{P}} w(\mathcal{P}) \Delta_{\mathcal{P}}
$$

and it follows that

$$
S\left(q_{1}, \ldots, q_{k} ; h\right)=\sum_{\mathcal{P}} w(\mathcal{P}) \sum_{\substack{a_{1}, \ldots, a_{k} \\ 1 \leq a_{i} \leq q_{i} \\\left(a_{i}, q_{i}\right)=1 \\ \sum a_{i} / q_{i} \in \mathbb{Z}}} \prod_{m=1}^{M} E\left(\sum_{i \in \mathcal{S}_{m}} \frac{a_{i}}{q_{i}}\right)
$$

where $\mathcal{P}=\left\{\mathcal{S}_{1}, \ldots, \mathcal{S}_{M}\right\}$

If there is a prime $p$ such that $p \mid q_{i}$ for exactly one $i$, then the condition $\sum a_{i} / q_{i} \in \mathbb{Z}$ cannot be fulfilled with $\left(a_{i}, q_{i}\right)=1$, so the sum (52) is empty, and hence $S\left(q_{1}, \ldots, q_{k} ; h\right)=$ 0 . We therefore assume that each prime dividing $\left[q_{1}, \ldots, q_{k}\right]$ divides at least two of the $q_{i}$.

To facilitate our discussion of various types of partitions, we introduce some notation. Let $\mathcal{M}=\{1, \ldots, M\}$. For a partition $\mathcal{P}=\left\{\mathcal{S}_{1}, \ldots, \mathcal{S}_{M}\right\}$, put

$$
\begin{array}{ll}
\mathcal{M}_{1}=\left\{m \in \mathcal{M}: \operatorname{card} \mathcal{S}_{m}=1\right\}, & m_{1}=\operatorname{card} \mathcal{M}_{1} ; \\
\mathcal{M}_{2}=\left\{m \in \mathcal{M}: \operatorname{card} \mathcal{S}_{m} \geq 2\right\}, & m_{2}=\operatorname{card} \mathcal{M}_{2} ; \\
\mathcal{N}_{1}=\bigcup_{\operatorname{card} \mathcal{S}_{m}=1} \mathcal{S}_{m}, & n_{1}=\operatorname{card} \mathcal{N}_{1} ; \\
\mathcal{N}_{2}=\bigcup_{\text {card } \mathcal{S}_{m} \geq 2} \mathcal{S}_{m}, & n_{2}=\operatorname{card} \mathcal{N}_{2} .
\end{array}
$$


Of course, $m_{1}+m_{2}=M, n_{1}+n_{2}=k$, and $m_{1}=n_{1}$. We first bound the contribution made by those partitions such that card $\mathcal{S}_{m} \geq 3$ for some $m$. For $m \in \mathcal{M}_{1}$ we use (27) to see that $\left|E\left(a_{i} / q_{i}\right)\right| \leq F\left(a_{i} / q_{i}\right)$. For $m \in \mathcal{M}_{2}$ we use the trivial bound

$$
\left|E\left(\sum_{i \in \mathcal{S}_{m}} \frac{a_{i}}{q_{i}}\right)\right| \leq h
$$

Hence

$$
\left|\sum_{\substack{a_{1}, \ldots, a_{k} \\ 1 \leq a_{i} \leq q_{i} \\\left(a_{i}, q_{i}\right)=1 \\ \sum a_{i} / q_{i} \in \mathbb{Z}}} \prod_{m=1}^{M} E\left(\sum_{i \in \mathcal{S}_{m}} \frac{a_{i}}{q_{i}}\right)\right| \leq h^{m_{2}} \sum_{\substack{a_{1}, \ldots, a_{k} \\ 1 \leq a_{i} \leq q_{i} \\\left(a_{i}, q_{i}\right)=1 \\ \sum a_{i} / q_{i} \in \mathbb{Z}}} \prod_{i \in \mathcal{N}_{1}} F\left(\frac{a_{i}}{q_{i}}\right)
$$

For $i \in \mathcal{N}_{1}$ we take $G_{i}(x)=F(x)$ for $0<x<1$, and $G_{i}(1)=0$. For $i \in \mathcal{N}_{2}$, we take $G_{i}(x)=1$ for all $x$. Thus by Lemma 1 we see that the above is

$$
\leq \frac{h^{m_{2}}}{\left[q_{1}, \ldots, q_{k}\right]} \prod_{i \in \mathcal{N}_{1}}\left(q_{i} \sum_{a=1}^{q_{i}-1} F\left(\frac{a}{q_{i}}\right)^{2}\right)^{1 / 2} \prod_{i \in \mathcal{N}_{2}}\left(q_{i} \sum_{a=1}^{q_{i}} 1\right)^{1 / 2}
$$

If $q \leq h$, then

$$
\sum_{a=1}^{q-1} F\left(\frac{a}{q}\right)^{2} \ll \sum_{0<a \leq q / 2}\left(\frac{q}{a}\right)^{2} \ll q^{2}
$$

If $q>h$, then

$$
\sum_{a=1}^{q-1} F\left(\frac{a}{q}\right)^{2} \ll \sum_{0<a \leq q / h} h^{2}+\sum_{q / h<a \leq q / 2}\left(\frac{q}{a}\right)^{2} \ll q h .
$$

Thus in any case,

$$
\sum_{a=1}^{q-1} F\left(\frac{a}{q}\right)^{2} \ll q \min (q, h) .
$$

Thus the expression (53) is

$$
\ll \frac{q_{1} \cdots q_{k}}{\left[q_{1}, \ldots, q_{k}\right]} h^{n_{1} / 2+m_{2}} .
$$

The $n_{2}$ members of $\mathcal{N}_{2}$ are partitioned into $m_{2}$ sets, each one containing at least two members, and at least one containing 3 or more numbers. Thus

$$
n_{2}=\sum_{m \in \mathcal{M}_{2}} \operatorname{card} S_{m} \geq 1+2 \sum_{m \in \mathcal{M}_{2}} 1=1+2 m_{2}
$$

and hence

$$
\frac{n_{1}}{2}+m_{2} \leq \frac{n_{1}+n_{2}-1}{2}=\frac{k-1}{2}
$$


We also observe that

$$
\begin{aligned}
\sum_{\substack{q_{1}, \ldots, q_{k} \\
q_{i} \mid Q}}\left(\prod_{i=1}^{k} \frac{\mu\left(q_{i}\right)^{2}}{\phi\left(q_{i}\right)}\right) \frac{q_{1} \cdots q_{k}}{\left[q_{1}, \ldots, q_{k}\right]} & \leq \sum_{d \mid Q} \frac{1}{d}\left(\sum_{q \mid d} \frac{q}{\phi(q)}\right)^{k}=\sum_{d \mid Q} \prod_{p \mid d}\left(1+\frac{p}{p-1}\right)^{k} \\
& =\prod_{p \mid Q}\left(1+\frac{1}{p}\left(1+\frac{p}{p-1}\right)^{k}\right) \ll_{k}(\log h)^{2^{k}} .
\end{aligned}
$$

Thus we have shown that

$$
\begin{aligned}
R_{k}(h)= & \sum_{\substack{\mathcal{P} \\
\operatorname{card} \mathcal{S}_{m} \leq 2}} w(\mathcal{P}) \sum_{\substack{q_{1}, \ldots, q_{k} \\
1<q_{i} \mid Q}} \prod_{i=1}^{k} \frac{\mu\left(q_{i}\right)}{\phi\left(q_{i}\right)} \sum_{\substack{a_{1}, \ldots, a_{k} \\
1 \leq a_{i} \leq q_{i} \\
\left(a_{i}, q_{i}\right)=1 \\
\sum a_{i} / q_{i} \in \mathbb{Z}}} \prod_{m=1}^{M} E\left(\sum_{i \in \mathcal{S}_{m}} \frac{a_{i}}{q_{i}}\right) \\
& +O\left(h^{(k-1) / 2+\varepsilon}\right) .
\end{aligned}
$$

Suppose that the partition $\mathcal{P}$ consists of $j$ doubleton sets and $k-2 j$ singleton sets. Since no other $\delta$-product is equivalent to $\Delta_{\mathcal{P}}$, and $\left|\Delta_{\mathcal{P}}\right|=j$, so $w(\mathcal{P})=(-1)^{j}$. The number of such partitions is

$$
\left(\begin{array}{c}
k \\
2 j
\end{array}\right) \frac{(2 j) !}{j ! 2^{j}}
$$

Since the $q_{i}$ are interchangeable, we multiply by the above factor, and restrict our attention to one such partition: doubletons $\{i, i+j\}$ for $1 \leq i \leq j$ and singletons $\{i\}$ for $2 j+1 \leq i \leq k$. Thus the main term in $(55)$ is

$$
\sum_{0 \leq j \leq k / 2}(-1)^{j}\left(\begin{array}{c}
k \\
2 j
\end{array}\right) \frac{(2 j) !}{j ! 2^{j}} \sum_{\substack{q_{1}, \ldots, q_{k} \\
1<q_{i} \mid Q}} \prod_{i=1}^{k} \frac{\mu\left(q_{i}\right)}{\phi\left(q_{i}\right)} \sum_{\substack{a_{1}, \ldots, a_{k} \\
1 \leq a_{i} \leq q_{i} \\
\left(a_{i}, q_{i}\right)=1 \\
\sum a_{i} / q_{i} \in \mathbb{Z}}} \prod_{i=1}^{j} E\left(\frac{a_{i}}{q_{i}}+\frac{a_{i+j}}{q_{i+j}}\right) \prod_{i=2 j+1}^{k} E\left(\frac{a_{i}}{q_{i}}\right) .
$$

For $1 \leq i \leq j$, let $b_{i}$ and $r_{i}$ be defined by the relations

$$
\frac{b_{i}}{r_{i}} \equiv \frac{a_{i}}{q_{i}}+\frac{a_{i+j}}{q_{i+j}} \quad(\bmod 1), \quad 1 \leq b_{i} \leq r_{i}, \quad\left(b_{i}, r_{i}\right)=1
$$

and put

$$
H\left(\frac{b}{r}\right)=E\left(\frac{b}{r}\right) \sum_{\substack{d_{1}, d_{2} \\ 1<d_{i} \mid Q}} \frac{\mu\left(d_{1}\right) \mu\left(d_{2}\right)}{\phi\left(d_{1}\right) \phi\left(d_{2}\right)} \sum_{\substack{c_{1}, c_{2} \\ 1 \leq c_{i} \leq d_{i} \\\left(c_{i}, d_{i}\right)=1 \\ c_{1} \\ d_{1}} \frac{c_{2}}{d_{2}} \equiv \frac{b}{r}(\bmod 1)} 1
$$


Then the sum over the $q_{i}$ in $(56)$ is

$$
\sum_{\substack{r_{1}, \ldots, r_{j} \\ r_{i} \mid Q}} \sum_{\substack{b_{1}, \ldots, b_{j} \\ 1 \leq b_{i} \leq r_{i} \\\left(b_{i}, r_{i}\right)=1}} \prod_{i=1}^{j} H\left(\frac{b_{i}}{r_{i}}\right) \sum_{\substack{q_{2 j+1}, \ldots, q_{k} \\ 1<q_{i} \mid Q}} \sum_{\substack{a_{2 j}+1, \ldots, a_{k} \\ 1 \leq a_{i} \leq q_{i} \\\left(a_{i}, q_{i}\right)=1 \\ \sum a_{i} / q_{i} \in \mathbb{Z}}} \prod_{i=2 j+1}^{k} \frac{\mu\left(q_{i}\right)}{\phi\left(q_{i}\right)} E\left(\frac{a_{i}}{q_{i}}\right) .
$$

We now separate those $i$ for which $r_{i}=1$ from those with $r_{i}>1$. Let $\ell$ denote the number of $i$ for which $r_{i}>1$. Since there are $\left(\begin{array}{l}j \\ \ell\end{array}\right)$ ways of choosing the $\ell$ values of the $i$ from $\{1, \ldots, j\}$, the above is

$$
\sum_{\ell=0}^{j}\left(\begin{array}{l}
j \\
\ell
\end{array}\right) H(1)^{j-\ell} M(\ell)
$$

where

$$
M(\ell)=\sum_{\substack{r_{1}, \ldots, r_{\ell} \\ 1<r_{i} \mid Q}} \sum_{\substack{b_{1}, \ldots, b_{\ell} \\ 1 \leq b_{i} \leq r_{i} \\\left(b_{i}, r_{i}\right)=1}} \prod_{i=1}^{\ell} H\left(\frac{b_{i}}{r_{i}}\right) \sum_{\substack{q_{2 j+1}, \ldots, q_{k} \\ 1<q_{i} \mid Q}} \sum_{\substack{a_{2 j+1}, \ldots, a_{k} \\ 1 \leq a_{i} \leq q_{i} \\\left(a_{i}, q_{i}\right)=1 \\ \sum a_{i} / q_{i}+\sum b_{i} / r_{i} \in \mathbb{Z}}} \prod_{i=2 j+1}^{k} \frac{\mu\left(q_{i}\right)}{\phi\left(q_{i}\right)} E\left(\frac{a_{i}}{q_{i}}\right) .
$$

We note that $M(0)=V_{2 k-j}(Q ; h)$.

Next we show that the contributions of $\ell>0$ can be absorbed in the error term. If there is a prime $p$ that divides exactly one of the numbers $r_{1}, \ldots, r_{\ell}, q_{2 j+1}, \ldots, q_{k}$, then the condition that $\sum a_{i} / q_{i}+\sum b_{i} / r_{i} \in \mathbb{Z}$ cannot be fulfilled with $\left(a_{i}, q_{i}\right)=\left(b_{i}, r_{i}\right)=1$, so the sum is empty, and the sum over the $q_{i}$ and $r_{i}$ vanishes. Thus we may restrict our attention to those choices of $q_{i}$ and $r_{i}$ for which every prime divisor of $d=\left[q_{2 j+1}, \ldots, q_{k}, r_{1}, \ldots, r_{\ell}\right]$ divides at least two of these numbers. Hence by Lemma 1,

$$
M(\ell) \ll \sum_{\substack{r_{1}, \ldots, r_{\ell} \\ 1<r_{i} \mid Q}} \sum_{\substack{q_{2 j+1, \ldots, q_{k}} \\ 1<q_{i} \mid Q}} \frac{1}{d} \prod_{i=1}^{\ell}\left(r_{i} \sum_{b=1}^{r_{i}-1}\left|H\left(\frac{b}{r_{i}}\right)\right|^{2}\right)^{1 / 2} \prod_{i=2 j+1}^{k}\left(\frac{q_{i}}{\phi\left(q_{i}\right)^{2}} \sum_{a=1}^{q_{i}-1} F\left(\frac{a}{q_{i}}\right)^{2}\right)^{1 / 2}
$$

In order to assess the above, we estimate $H(b / r)$. We note that $H(b / r)=0$ if $r \nmid Q$. Thus we suppose that $r \mid Q$, and that $r>1$. For $i=1,2$ we write $d_{i}=s_{i} t_{i}$ where $s_{i} \mid r$, $t_{i} \mid Q / r$. By the Chinese Remainder Theorem there exist unique $e_{i}\left(\bmod s_{i}\right)$ and $f_{i}(\bmod$ $t_{i}$ ) such that

$$
\frac{e_{i}}{s_{i}}+\frac{f_{i}}{t_{i}} \equiv \frac{c_{i}}{d_{i}} \quad(\bmod 1), \quad\left(e_{i}, s_{i}\right)=\left(f_{i}, t_{i}\right)=1 .
$$

From the conditions $c_{1} / d_{1}+c_{2} / d_{2} \equiv b / r(\bmod 1),(b, r)=1$ it follows that

$$
\frac{e_{1}}{s_{1}}+\frac{e_{2}}{s_{2}} \equiv \frac{b}{r} \quad(\bmod 1), \quad\left[s_{1}, s_{2}\right]=r, \quad t_{1}=t_{2}, \quad f_{1}+f_{2} \equiv 0 \quad\left(\bmod t_{1}\right)
$$


Put $t=t_{1}=t_{2}$. Hence

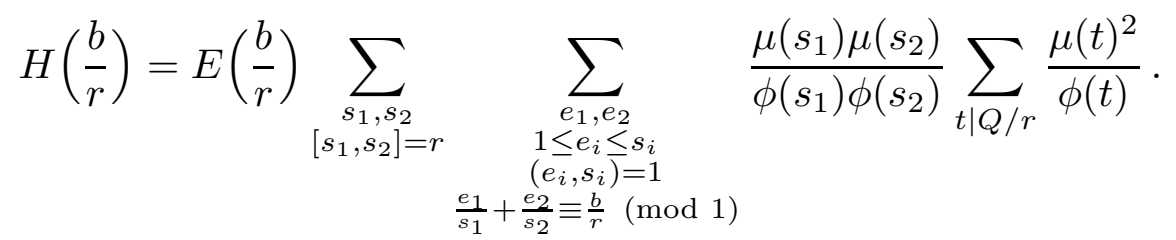

For given $s_{1}, s_{2}$, the number of pairs $e_{1}, e_{2}$ with the required properties is $\leq \phi\left(\left(s_{1}, s_{2}\right)\right)=$ $\phi\left(s_{1}\right) \phi\left(s_{2}\right) / \phi(r)$. Hence

$$
\begin{aligned}
H\left(\frac{b}{r}\right) & \ll F\left(\frac{b}{r}\right) \sum_{\substack{s_{1}, s_{2} \\
\left[s_{1}, s_{2}\right]=r}} \frac{1}{\phi(r)} \prod_{p \mid Q / r}\left(1+\frac{1}{p-1}\right)=F\left(\frac{b}{r}\right) \frac{3^{\omega(r)}}{r} \prod_{p \mid Q}\left(1+\frac{1}{p-1}\right) \\
& \ll F\left(\frac{b}{r}\right) \frac{3^{\omega(r)}}{r} \log h .
\end{aligned}
$$

By (54) it follows that

$$
\sum_{b=1}^{r-1}\left|H\left(\frac{b}{r}\right)\right|^{2} \ll \frac{h}{r} 9^{\omega(r)}(\log h)^{2} .
$$

On inserting this and (54) in (59), we find that

$$
\begin{aligned}
M(\ell) & \ll \sum_{\substack{r_{1}, \ldots, r_{\ell} \\
r_{i} \mid Q}} \sum_{\substack{q_{2 j+1} \ldots, q_{k} \\
q_{i} \mid Q}} \frac{1}{d} \prod_{i=1}^{\ell}\left(h^{1 / 2} 3^{\omega\left(r_{i}\right)} \log h\right) \prod_{i=2 j+1}^{k} \frac{h^{1 / 2} q_{i}}{\phi\left(q_{i}\right)} \\
& \ll h^{(k-2 j+\ell) / 2}(\log h)^{\ell} \sum_{d \mid Q} \frac{1}{d}\left(\sum_{r \mid d} 3^{\omega(r)}\right)^{\ell}\left(\sum_{q \mid d} \frac{q}{\phi(q)}\right)^{k-2 j} \\
& \leq h^{(k-2 j+\ell) / 2}(\log h)^{\ell} \prod_{p \mid Q}\left(1+\frac{4^{k-2 j+\ell}}{p}\right) \\
& \ll h^{(k-2 j+\ell) / 2+\varepsilon} .
\end{aligned}
$$

From (57) we see that

$$
H(1)=h \sum_{\substack{d \mid Q \\ d>1}} \frac{\mu(q)^{2}}{\phi(q)}
$$

Hence the expression (58) is

$$
\left(h \sum_{\substack{d \mid Q \\ d>1}} \frac{\mu(q)^{2}}{\phi(q)}\right)^{j} V_{k-2 j}(Q ; h)+O\left(h^{(k-1) / 2+\varepsilon}\right) .
$$


On inserting this in (56), we find that

$$
R_{k}(h)=\sum_{0 \leq j \leq k / 2}\left(\begin{array}{c}
k \\
2 j
\end{array}\right) \frac{(2 j) !}{j ! 2^{j}}\left(-h \sum_{\substack{d \mid Q \\
d>1}} \frac{\mu(d)^{2}}{\phi(d)}\right)^{j} V_{k-2 j}(Q ; h)+\left(h^{(k-1) / 2+\varepsilon}\right) .
$$

We are at last prepared to make our appeal to Theorem 1 . If $k$ is odd then $k-2 j$ is odd, so there is no main term. Suppose that $k$ is even. Then the main term is

$$
\begin{aligned}
& \sum_{0 \leq j \leq k / 2}\left(\begin{array}{c}
k \\
2 j
\end{array}\right) \frac{(2 j) !}{j ! 2^{j}}\left(-h \sum_{\substack{d \mid Q \\
d>1}} \frac{\mu(d)^{2}}{\phi(d)}\right)^{j} \frac{(k-2 j) !}{(k / 2-j) ! 2^{k / 2-j}} V_{2}(Q ; h)^{k / 2-j} \\
= & \frac{k !}{(k / 2) ! 2^{k / 2}} \sum_{j=0}^{k / 2}\left(\begin{array}{c}
k / 2 \\
j
\end{array}\right) V_{2}(Q ; h)^{k / 2-j}\left(-h \sum_{\substack{d \mid Q \\
d>1}} \frac{\mu(d)^{2}}{\phi(d)}\right)^{j} \\
= & \mu_{k}\left(V_{2}(Q ; h)-h \sum_{\substack{d \mid Q \\
d>1}} \frac{\mu(d)^{2}}{\phi(d)}\right)^{k / 2}
\end{aligned}
$$

by the binomial theorem.

By (49) we see that the above is

$$
\mu_{k}(-h \log h+A h)^{k / 2}+O\left(h^{(k-1) / 2+\varepsilon}\right) .
$$

This gives the stated result.

\section{Proof of Theorem 3}

Clearly $M_{0}(N ; H)=N$. Thus we have the case $K=0$ of $(21)$ unconditionally, and with no error term. It is also convenient to dispose of the case $K=1$ before proceeding to the main argument. Since $\mathfrak{S}(\{h\})=1$, it follows from our hypothesis (20) that $\sum_{n=1}^{N} \Lambda(n+$ $h)=N+O\left(N^{1 / 2+\varepsilon}\right)$. Hence $\sum_{n=1}^{N} \Lambda_{0}(n+h) \ll N^{1 / 2+\varepsilon}$, and thus by (19) we see that $M_{1}(N ; H) \ll H N^{1 / 2+\varepsilon}$, which suffices.

From now on we assume that $K$ is fixed, $K \geq 2$, and we ignore possible dependence of implicit contstants on $K$. Let $\mathcal{D}=\left\{d_{1}, \ldots, d_{k}\right\}$ be a set of $k$ distinct integers with $1 \leq d_{i} \leq H$ for $1 \leq i \leq k$. Suppose that the $h_{i}$ in (19) take the values $d_{i}$ with multiplicities $M_{i}$. Then the right hand side of (19) is

$$
=\sum_{k=1}^{K} \sum_{\substack{M_{1}, \ldots, M_{k} \\
M_{i} \geq 1 \\
\sum M_{i}=K}}\left(\begin{array}{c}
K \\
M_{1} \cdots M_{k}
\end{array}\right) \frac{1}{k !} \sum_{\substack{d_{1}, \ldots, d_{k} \\
1 \leq d_{i} \leq H \\
d_{i} \text { distinct }}} \sum_{n=1}^{N} \prod_{i=1}^{k} \Lambda_{0}\left(n+d_{i}\right)^{M_{i}} .
$$

Here the $1 / k$ ! is necessary because any permutation of $d_{1}, \ldots, d_{k}$ gives rise to the same set $\mathcal{D}$. For positive integers $m$ we put $\Lambda_{m}(n)=\Lambda(n)^{m} \Lambda_{0}(n)$. If $M \geq 1$, then by the binomial theorem

$$
\Lambda_{0}(n)^{M}=\Lambda_{0}(n)(\Lambda(n)-1)^{M-1}=\sum_{m=0}^{M-1}(-1)^{M-m-1}\left(\begin{array}{c}
M-1 \\
m
\end{array}\right) \Lambda_{m}(n) .
$$


On inserting this in (61), we find that

$$
\begin{aligned}
M_{K}(N ; H)=\sum_{k=1}^{K} \frac{1}{k !} \sum_{\substack{M_{1}, \ldots, M_{k} \\
M_{i} \geq 1 \\
\sum M_{i}=K}}\left(\begin{array}{c}
K \\
M_{1} \cdots M_{k}
\end{array}\right) \\
\quad \times \sum_{\substack{m_{1}, \ldots, m_{k} \\
0 \leq m_{i}<M_{i}}} \prod_{i=1}^{k}(-1)^{M_{i}-1-m_{i}}\left(\begin{array}{c}
M_{i}-1 \\
m_{i}
\end{array}\right) L_{k}(\boldsymbol{m})
\end{aligned}
$$

where

$$
L_{k}(\boldsymbol{m})=\sum_{\substack{d_{1}, \ldots, d_{k} \\ 1 \leq d_{i} \leq H \\ d_{i} \text { distinct }}} \sum_{n=1}^{N} \prod_{i=1}^{k} \Lambda_{m_{i}}\left(n+d_{i}\right)
$$

To estimate the $L_{k}(\boldsymbol{m})$, we must distinguish between those $i$ for which $m_{i}=0$ and those for which $m_{i}>0$. To this end we set $\mathcal{K}=\{1, \ldots k\}$, and introduce the following notation:

$$
\begin{aligned}
\mathcal{H} & =\left\{i \in \mathcal{K}: m_{i} \geq 1\right\}, & h & =\operatorname{card} \mathcal{H} ; \\
\mathcal{J} & =\left\{i \in \mathcal{K}: m_{i}=0\right\}, & k-h & =\operatorname{card} \mathcal{J} ; \\
\mathcal{J} & \subseteq \mathcal{K}, & j & =\operatorname{card} \mathcal{J} .
\end{aligned}
$$

Thus

$$
\begin{aligned}
\prod_{i \in \mathcal{J}} \Lambda_{0}\left(n+d_{i}\right) \prod_{i \in \mathcal{H}} \Lambda\left(n+d_{i}\right) & =\prod_{i \in \mathcal{J}} \Lambda_{0}\left(n+d_{i}\right) \prod_{i \in \mathcal{H}}\left(\Lambda_{0}\left(n+d_{i}\right)+1\right) \\
& =\sum_{\substack{\mathcal{J} \\
\mathcal{J} \subseteq \mathcal{J} \subseteq \mathcal{K}}} \prod_{i \in \mathcal{J}} \Lambda_{0}\left(n+d_{i}\right) .
\end{aligned}
$$

From our hypothesis (20) it follows that

$$
\sum_{n \leq x} \prod_{i \in \mathcal{J}} \Lambda_{0}\left(n+d_{i}\right) \prod_{i \in \mathcal{H}} \Lambda\left(n+d_{i}\right)=x \sum_{\substack{\mathcal{J} \\ \mathcal{J} \subseteq \mathcal{J} \subseteq \mathcal{K}}} \mathfrak{S}_{0}\left(\mathcal{D}_{\mathfrak{J}}\right)+O\left(N^{1 / 2+\varepsilon}\right)
$$

uniformly for $0 \leq x \leq N$ where $\mathcal{D}_{\mathfrak{J}}=\left\{d_{i}: i \in \mathfrak{J}\right\}$. With $\boldsymbol{m}=\left(m_{1}, \ldots, m_{k}\right)$ fixed for the moment, write the above briefly as $f(x)=c x+O\left(N^{1 / 2+\varepsilon}\right)$. Then

$$
\begin{aligned}
\sum_{n=1}^{N} & \left(\prod_{i \in \mathcal{J}} \Lambda_{0}\left(n+d_{i}\right)\right)\left(\prod_{i \in \mathcal{H}} \Lambda\left(n+d_{i}\right)\left(\log \left(n+d_{i}\right)\right)^{m_{i}-1}\left(\log \left(n+d_{i}\right)-1\right)\right) \\
& =\int_{1^{-}}^{N} \prod_{i \in \mathcal{H}}\left(\log \left(x+d_{i}\right)\right)^{m_{i}-1}\left(\log \left(x+d_{i}\right)-1\right) d f(x),
\end{aligned}
$$


which by integration by parts is

$$
=c \int_{1}^{N} \prod_{i \in \mathcal{H}}\left(\log \left(x+d_{i}\right)\right)^{m_{i}-1}\left(\log \left(x+d_{i}\right)-1\right) d x+O\left(N^{1 / 2+\varepsilon}\right) .
$$

For $m>0, \Lambda_{m}(n)$ is nonzero only when $n$ is a primepower, and

$$
\Lambda_{m}(n)=\Lambda(n)(\log n)^{m-1}(\log n-1)
$$

if $n$ is prime. Thus if $n$ is an integer such that $n+d_{i}$ is prime for all $i \in \mathcal{H}$, then the summand in (64) is

$$
\prod_{i=1}^{k} \Lambda_{m_{i}}\left(n+d_{i}\right)
$$

Those $n$ for which $n+d_{i}$ is a higher power of a prime for one or more $i \in \mathcal{H}$ contribute an amount $\ll N^{1 / 2+\varepsilon}$ to the sum (64). Thus the sum (64) is

$$
=\sum_{n=1}^{N} \prod_{i=1}^{k} \Lambda_{m_{i}}\left(n+d_{i}\right)+O\left(N^{1 / 2+\varepsilon}\right) .
$$

Next we approximate the integral in (65) by a similar integral that is independent of the $d_{i}$. First we note that if $k=K$, then $M_{i}=1$ for all $i$, and hence $m_{i}=0$ for all $i$, so that $h=0$. Thus we may suppose that $k<K$. It is useful to note that

$$
\sum_{i \in \mathcal{H}} m_{i}=\sum_{i=1}^{k} m_{i} \leq \sum_{i=1}^{k}\left(M_{i}-1\right)=K-k .
$$

If $x \geq 1$ and $1 \leq d \leq H$, then

$$
\log (x+d)=\log x+O(d / x)=\log x+O(H / x) .
$$

Thus the integrand is

$$
\prod_{i \in \mathcal{H}}\left((\log x)^{m_{i}-1}(\log x-1)\right)+O\left(H x^{-1}(\log N)^{K-k-1}\right)
$$

and so the integral is $I_{\boldsymbol{m}}(N)+O\left(H(\log N)^{K-k}\right)$ where

$$
I_{\boldsymbol{m}}(N)=\int_{1}^{N} \prod_{i \in \mathcal{H}}\left((\log x)^{m_{i}-1}(\log x-1)\right) d x .
$$

On assembling our estimates, we find that

$$
\sum_{n=1}^{N} \prod_{i=1}^{k} \Lambda_{m_{i}}\left(n+d_{i}\right)=\left(\sum_{\substack{\mathcal{J} \\ \mathcal{J} \subseteq \mathcal{J} \subseteq \mathcal{K}}} \mathfrak{S}_{0}\left(\mathcal{D}_{\mathfrak{J}}\right)\right)\left(I_{\boldsymbol{m}}(N)+O\left(H(\log N)^{K-k}\right)\right)+O\left(N^{1 / 2+\varepsilon}\right) .
$$


By (43) we see that the first error term is $\ll H(\log N)^{K} \ll N^{1 / K+\varepsilon} \ll N^{1 / 2+\varepsilon}$, since $H \leq N^{1 / K}$ and $K \geq 2$.

On summing both sides of the above over all choices of distinct $d_{i}$, we find that

$$
L_{k}(\boldsymbol{m})=I_{\boldsymbol{m}}(N) \sum_{\substack{\mathcal{J} \\ \mathcal{I} \subseteq \mathcal{J} \subseteq \mathcal{K} \mathcal{K}}} \sum_{\substack{d_{1}, \ldots, d_{k} \\ 1 \leq d_{i} \leq H \\ d_{i}} \text { distinct }} \mathfrak{S}_{0}\left(\mathcal{D}_{\mathfrak{J}}\right)+O\left(H^{k} N^{1 / 2+\varepsilon}\right) .
$$

Once the $d_{i}$ have been chosen for $i \in \mathcal{J}$, there are $(H-j)(H-j-1) \cdots(H-k+1)$ ways of choosing the remaining $d_{i}$. Hence the above is

$$
=I_{\boldsymbol{m}}(N) \sum_{\substack{\mathcal{J} \\ \mathcal{J} \subseteq \mathcal{J} \subseteq \mathcal{K}}} R_{j}(H)(H-j) \cdots(H-k+1)+O\left(H^{k} N^{1 / 2+\varepsilon}\right) .
$$

The product in the sum is $H^{k-j}+O\left(H^{k-j-1}\right)$. By Theorem 2 we know that $R_{j}(H) \ll$ $(H \log H)^{j / 2}$, and using this we see that (isolating the term $\mathcal{J}=\mathcal{J}$ )

$$
\begin{aligned}
\sum_{\substack{\mathcal{J} \\
\mathcal{J} \subseteq \mathcal{J} \subseteq \mathcal{K}}} R_{j}(H)(H-j) & \cdots(H-k+1) \\
& =R_{k-h}(H)\left(H^{h}+O\left(H^{h-1}\right)\right)+O\left(\sum_{k-h+1 \leq j \leq k}(H \log H)^{j / 2} H^{k-j}\right) \\
& =R_{k-h}(H) H^{h}+O\left((H \log H)^{(k-h+1) / 2} H^{h-1}\right) .
\end{aligned}
$$

Therefore

$$
L_{k}(\boldsymbol{m})=I_{\boldsymbol{m}}(N)\left(R_{k-h}(H) H^{h}+O\left((H \log H)^{(k-h+1) / 2} H^{h-1}\right)\right)+O\left(H^{k} N^{1 / 2+\varepsilon}\right) .
$$

We insert the above in (62). In assessing the sizes of the various terms, it is useful to note that

$$
K=\sum_{i=1}^{k} M_{i}=\sum_{i \in \mathcal{H}} M_{i}+\sum_{i \in \mathcal{J}} M_{i} \geq 2 \operatorname{card} \mathcal{H}+\operatorname{card} \mathcal{J}=2 h+(k-h)=h+k .
$$

By (67) we see that

$$
I_{\boldsymbol{m}}(N) \sim N(\log N)^{\sum_{i \in \mathcal{H}} m_{i}} \ll N(\log N)^{K-k} .
$$

First we show that terms for which $h+k<K$ contribute a negligible amount to (62). Since $R_{k-h}(H) \ll(H \log H)^{(k-h) / 2}$ by Theorem 2 , it follows from (68), (69), and (70) that

$$
\begin{aligned}
L_{k}(\boldsymbol{m}) & \ll N(\log N)^{K-k}(H \log H)^{(k-h) / 2} H^{h}+H^{k} N^{1 / 2+\varepsilon} \\
& \ll N(\log N)^{K}\left(\frac{H}{\log N}\right)^{(k+h) / 2}\left(\frac{\log H}{\log N}\right)^{(k-h) / 2}+H^{K} N^{1 / 2+\varepsilon} .
\end{aligned}
$$


Thus the contribution of these terms to $(62)$ is

$$
\ll N(\log N)^{K}\left(\frac{H}{\log N}\right)^{(K-1) / 2}+H^{K} N^{1 / 2+\varepsilon} .
$$

Finally we consider those terms in (62) for which $h+k=K$. Since $h \leq k$, it follows that $k \geq K / 2$. We also have card $\mathcal{H}=h=K-k$, and card $\mathcal{J}=k-h=2 k-K$. Since equality holds in (69), it follows that $M_{i}=2$ for all $i \in \mathcal{H}$ and that $M_{i}=1$ for all $i \in \mathcal{J}$. Thus $m_{i}=M_{i}-1$ for all $i$, and for such $\boldsymbol{m}$ we have

say. Hence

$$
I_{\boldsymbol{m}}(N)=\int_{1}^{N}(\log x-1)^{K-k} d x=I_{K-k}(N)
$$

$$
\begin{aligned}
L_{k}(\boldsymbol{m})=I_{K-k}(N) R_{2 k-K}(H) H^{K-k} & +O\left(N(\log N)^{K-k}(H \log H)^{(2 k-K+1) / 2} H^{K-k-1}\right) \\
& +O\left(H^{k} N^{1 / 2+\varepsilon}\right) \\
=I_{K-k}(N) R_{2 k-K}(H) H^{K-k} & +O\left(N(\log N)^{K}\left(\frac{H}{\log N}\right)^{(K-1) / 2}\right)+O\left(H^{K} N^{1 / 2+\varepsilon}\right) .
\end{aligned}
$$

Once $k$ is selected, there are precisely $\left(\begin{array}{c}k \\ K-k\end{array}\right)$ ways of choosing the set $\mathcal{H}$, and hence using (71) and the above,

$$
\begin{aligned}
M_{K}(N ; H)= & \sum_{K / 2 \leq k \leq K} \frac{K !}{k ! 2^{K-k}}\left(\begin{array}{c}
k \\
K-k
\end{array}\right) I_{K-k}(N) R_{2 k-K}(H) H^{K-k} \\
& +O\left(N(\log N)^{K}\left(\frac{H}{\log N}\right)^{(K-1) / 2}\right)+O\left(H^{K} N^{1 / 2+\varepsilon}\right) .
\end{aligned}
$$

Suppose that $K$ is odd. Then so also is $2 k-K$, and hence by Theorem 2 the main terms in (72) are

$$
\ll N(\log N)^{K / 2} H^{K / 2-1 /(7 K)+\varepsilon} .
$$

Thus in this case

$$
\begin{aligned}
M_{K}(N ; H) & \ll N(\log N)^{K / 2} H^{K / 2-1 /(7 K)+\varepsilon}+N(\log N)^{K}\left(\frac{H}{\log N}\right)^{(K-1) / 2}+H^{K} N^{1 / 2+\varepsilon} \\
& \ll N(\log N)^{K / 2} H^{K / 2}\left(\frac{H}{\log N}\right)^{-1 /(8 K)}+H^{K} N^{1 / 2+\varepsilon} .
\end{aligned}
$$

Suppose that $K$ is even. By Theorem 2 it follows that

$$
\begin{gathered}
M_{K}(N ; H)=H^{K / 2} \sum_{k=K / 2}^{K} \frac{K !}{k ! 2^{K-k}}\left(\begin{array}{c}
k \\
K-k
\end{array}\right) \mu_{2 k-K} I_{K-k}(N)(-\log H+A)^{k-K / 2} \\
+O\left(N(\log N)^{K / 2} H^{K / 2}\left(\frac{H}{\log N}\right)^{-1 /(8 K)}+H^{K} N^{1 / 2+\varepsilon}\right) .
\end{gathered}
$$

Since $\mu_{k}=k ! /\left((k / 2) ! 2^{k / 2}\right)$ when $k$ is even, on writing $k=K / 2+\ell$ we see that the main term above is

$$
\mu_{K} H^{K / 2} \sum_{\ell=0}^{K / 2}\left(\begin{array}{c}
K / 2 \\
\ell
\end{array}\right) I_{K / 2-\ell}(N)(-\log H+A)^{\ell} .
$$

On taking the sum inside the integral, we obtain the stated result by the binomial theorem. 


\section{Heuristics in the manner of Hardy \& Littlewood}

The reasoning here is conducted in the manner that Hardy \& Littlewood [10] used to formulate their quantitative version of the prime $k$-tuple hypothesis. Let

$$
S(\alpha)=\sum_{n=1}^{N} \Lambda(n) e(n \alpha)
$$

By the prime number theorem for arithmetic progressions we know that if $(a, q)=1$, then $S(a / q) \sim \mu(q) N / \phi(q)$ provided that $q$ is not too large as a function of $N$. By partial summation it follows that $S(\alpha) \sim \mu(q) M(\alpha-a / q) / \phi(q)$ for $\alpha$ near $a / q$, where $M(\beta)=\sum_{n=1}^{N} e(n \beta)$. Put $E(\alpha)=\sum_{m=1}^{h} e(m \alpha)$, as in the proof of Theorem 1. If $h$ is small compared with $N$, then $S(\alpha) E(-\alpha)$ is approximately

$$
\sum_{n=1}^{N}\left(\sum_{m=1}^{h} \Lambda(m+n)\right) e(n \alpha)
$$

Moreover, when $\alpha$ is near $a / q, E(-\alpha)$ is approximately $E(-a / q)$. Thus when $\alpha$ is a number for which the expression above is large, we expect that that it is approximately

$$
\sum_{1 \leq q \leq N} \frac{\mu(q)}{\phi(q)} \sum_{\substack{a=1 \\(a, q)=1}}^{q} E(-a / q) M(\alpha-a / q) .
$$

We subtract the contribution of the term $a=q=1$ from both sides to see that

$$
\sum_{n=1}^{N}\left(\sum_{m=1}^{h} \Lambda(m+n)-h\right) e(n \alpha) \doteqdot \sum_{1<q \leq N} \frac{\mu(q)}{\phi(q)} \sum_{\substack{a=1 \\(a, q)=1}}^{q} E(a / q) M(\alpha-a / q) .
$$

Let $F(\alpha)$ denote the left hand side above. The $k$-fold convolution of $F$ with itself is

$$
\sum_{n=1}^{N}\left(\sum_{m=1}^{h} \Lambda(m+n)-h\right)^{k} e(n \alpha)=\int_{\sum \alpha_{i}=\alpha(\bmod 1)} \ldots \prod_{i=1}^{k} F\left(\alpha_{i}\right) d \alpha_{1} \cdots d \alpha_{k} .
$$

We set $\alpha=0$, and follow Hardy \& Littlewood in assuming that the main term arises by the alignment of the peaks in the multiple integral on the right hand side. Thus we expect that

$$
\sum_{n=1}^{N}\left(\sum_{m=1}^{h} \Lambda(m+n)-h\right)^{k} \sim N \sum_{\substack{q_{1}, \ldots, q_{k} \\ 1<q_{i} \leq N}}\left(\prod_{i=1}^{k} \frac{\mu\left(q_{i}\right)}{\phi\left(q_{i}\right)}\right) \sum_{\substack{a_{1}, \ldots, a_{k} \\ 1 \leq a_{i} \leq q_{i} \\\left(a_{i}, q_{i}\right)=1 \\ \sum a_{i} / q_{i} \in \mathbb{Z}}} \prod_{i=1}^{k} E\left(a_{i} / q_{i}\right) .
$$


We note that $|E(\beta)| \asymp h$ if $\|\beta\| \leq 1 / h$, and that $E(\beta) \ll 1 /\|\beta\|$ if $\|\beta\| \geq 1 / h$. The asymptotic size of the right hand side above could be determined by using the techniques used to prove Theorem 1. At this point we are content to argue more informally. If $k$ is odd then the terms do not make a very significant contribution. On the other hand, when $k$ is even, we find 'diagonal terms' in which the $q_{i}$ are equal in pairs, with the corresponding $a_{i}$ being the negatives of each other. The pairings can be made in

$$
(k-1)(k-3) \cdots 3 \cdot 1=\mu_{k}
$$

ways, so the contribution of these terms is

$$
\mu_{k} N \sum_{\substack{q_{1}, \ldots, q_{k / 2} \\ 1<q_{i} \leq N}} \prod_{i=1}^{k / 2}\left(\frac{\mu\left(q_{i}\right)^{2}}{\phi\left(q_{i}\right)^{2}} \sum_{\substack{a_{i}=1 \\\left(a_{i}, q_{i}\right)=1}}^{q_{i}}\left|E\left(a_{i} / q_{i}\right)\right|^{2}\right)=\mu_{k} N\left(\sum_{1<q \leq N} \frac{\mu(q)^{2}}{\phi(q)^{2}} \sum_{\substack{a=1 \\(a, q)=1}}^{q}|E(a / q)|^{2}\right)^{k / 2}
$$

If there are further equalities among the $q_{i}$ beyond this pairing, then the combinatorics must be adjusted, but such configurations contribute a lesser amount. Likewise, that the nondiagonal terms contribute a lesser amount can be demonstrated by using the techniques we used to prove Theorem 1. If $q<h$ then the inner sum above is $\ll \sum_{0<a<q}\|a / q\|^{-2} \ll q^{2}$, but if $q \geq h$ then the inner sum is approximately $h \phi(q)$. Since

$$
\sum_{q \leq y} \frac{\mu(q)^{2}}{\phi(q)}=\log y+O(1)
$$

it follows that the expression to be estimated is

$$
\sim \mu_{k} N\left(h \log \frac{N}{h}\right)^{k / 2}
$$

which supports the Conjecture.

\section{REFERENCES}

1. E. B. Bogomolny \& J. P. Keating, Random matrix theory and the Riemann zeros. II. n-point correlations, Nonlinearity 9 (1996), 911-935.

2. R. P. Brent, Irregularities in the distribution of primes and twin primes, Math. Comp. 29 (1975), 43-56; Correction 30 (1976), 198.

3. T. H. Chan, Pair correlation and distribution of prime numbers, PhD dissertation, University of Michigan, Ann Arbor, 2002, pp. vi+101.

4. H. Cramér, On the order of magnitude of the difference between consecutive prime numbers, Acta Arith. 2 (1936), 23-46.

5. P. J. Forrester \& A. M. Odlyzko, Gaussian unitary ensemble eigenvalues and Riemann $\zeta$ function zeros: a nonlinear equation for a new statistic, Phys. Rev. E (3) 54 (1996), R4493-R4495.

6. P. X. Gallagher, On the distribution of primes in short intervals, Mathematika 23 (1976), 4-9, Corrigendum, 28 (1981), 86.

7. D. A. Goldston, Linnik's theorem on Goldbach numbers in short intervals, Glasgow Math. J. 32 (1990), 285-297. 
8. D. A. Goldston \& H. L. Montgomery, On pair correlations of zeros and primes in short intervals, Analytic Number Theory and Diophantine Problems (Stillwater, OK, July 1984) (A. C. Adolphson, J. B. Conrey, A. Ghosh, R. I. Yager, eds.), Prog. Math. 70, Birkäuser, Boston, 1987, pp. 183-203.

9. A. Granville \& K. Soundararajan, An uncertainty principle for arithmetic sequences, preprint, available from www.arxiv.org.

10. G. H. Hardy \& J. E. Littlewood, Some problems of "Partitio Numerorum" (III): On the expression of a number as a sum of primes, Acta Math. 44 (1922), 1-70.

11. M. Hausman \& H. N. Shapiro, On the mean square distribution of primitive roots of unity, Comm. Pure App. Math. 26 (1973), 539-547.

12. H. Maier, Primes in short intervals, Michigan Math. J. 32 (1985), 221-225.

13. H. L. Montgomery, The pair correlation of zeros of the zeta function, Analytic Number Theory (St. Louis Univ., 1972), Proc. Sympos. Pure Math. 24, Amer. Math. Soc., Providence, 1973, pp. 181-193.

14. - Ten lectures on the interface between analytic number theory and harmonic analysis, CBMS 84, Amer. Math. Soc., Providence, 1994, pp. xii+220.

15. H. L. Montgomery \& K. Soundararajan, Beyond pair correlation, Paul Erdős and his Mathematics. I, Math. Studies 11, Bolyai Society, Budapest, 2002, pp. 507-514.

16. H. L. Montgomery \& R. C. Vaughan, On the distribution of reduced residues, Annals of Math. 123 (1986), 311-333.

17. _ A basic inequality, Congress in Number Theory (Zarautz, 1984), Universidad del País Vasco, Bilbao, 1989, pp. 163-175.

18. A. M. Odlyzko, On the distribution of spacings between zeros of the zeta function, Math. Comp. $\mathbf{4 8}$ (1987), 273-308.

19. E. M. Rains, High powers of random elements of compact Lie groups, Probab. Theory Related Fields 107 (1997), 219-241.

20. A. Selberg, On the normal density of primes in short intervals, and the difference between consecutive primes, Collected papers (Volume I), Springer, 1989, pp. 160-178.

Dept. of Math., Univ. of Michigan, Ann Arbor, Mi 48109-1109, USA email: hlm@umich.edu

Dept. of Math., Univ. of Michigan, Ann Arbor, MI 48109-1109, USA email: ksound@umich.edu 